\title{
Recent advances in all-in-one flexible supercapacitors
}

\author{
Tiezhu Guo ${ }^{1}$, Di Zhou ${ }^{1,2^{*}}$, Wenfeng $\mathrm{Liu}^{2}$ and Jinzhan $\mathrm{Su}^{3}$
}

\begin{abstract}
The rise of personalized flexible electronics has promoted the rapid development of flexible supercapacitors due to their long service life, fast charging-discharging rates and safe operation. Different from the traditional flexible supercapacitors, the all-in-one integrated flexible supercapacitors are more resistant to deformation and lower interface resistance, which have a broader application prospect in the field of flexible electronics. This review briefly summarizes the preparation methods and electrochemical properties of some typical all-in-one supercapacitors, represented by planar and fibrous structures in recent years. Firstly, the basic understanding of traditional flexible supercapacitors and all-in-one integrated flexible supercapacitors, and the key parameters of the performance of flexible supercapacitors are introduced. Subsequently, the hydrogel matrix all-in-one supercapacitors with different functional characteristics (stretchable, self-healing and compressible) and the nonhydrogel matrix (separator, flexible membrane) all-in-one supercapacitors are discussed. Furthermore, the challenges and future development of flexible supercapacitors are considered.
\end{abstract}

Keywords: flexible supercapacitors, all-in-one, hydrogel

\section{INTRODUCTION}

The exploration of clean and renewable energy, such as wind, solar and tidal power, is an effective way to solve the energy crisis. The development of energy storage devices is desired because wind and solar energy are highly dependent on geographical and natural conditions, resulting in unstable energy output. Energy storage devices can be divided into traditional electrostatic capacitor, chemical battery and supercapacitor (SC) [1].
Traditional capacitor is marked by high power density and low energy density, whereas chemical battery is characterized by high energy density and low power density [2]. SC is a type of energy storage device between traditional capacitor and chemical battery. It has attracted much attention due to its higher power density, faster charging-discharging rates and longer service life than the traditional batteries $[1,3]$. The booming development of wearable intelligent electronics has driven the demand for flexible electronic energy storage devices, such as electronic skin [4], health monitoring bioelectronics [5], biosensor [6], wearable smart textile [7], and flexible displays [8], which are extremely popular $[9,10]$. As research continues to deepen and diversify, some functional SCs have been designed. It is generally believed that flexible SCs (FSCs) need to have basic flexible mechanical properties (e.g., one or more kinds of foldable, bendable, stretchable, compressible, etc.).

On account of the device structure, FSCs can be divided into all-in-one and non-all-in-one devices [11]. Non-allin-one FSCs are made by pre-preparing electrode, electrolyte, separator, and then assembled into sandwich structures with distinct stacks. The all-in-one FSCs mainly integrate the electrode, electrolyte, separator and even the current collector on a flexible substrate, which not only dramatically reduces the interlayer interface resistance, but also avoids the use of non-active substance, effectively improving the device performance. Usually, flexible substrates are used as separator, such as hydrogels, polypropylene membrane, melamine foam, graphene oxide (GO) film, etc. In addition, flexible substrates are used only as a flexible substrate in the micro-SCs (MSCs). The conventional non-all-in-one FSCs are a sandwich

\footnotetext{
${ }^{1}$ Electronic Materials Research Laboratory, Key Laboratory of the Ministry of Education \& International Center for Dielectric Research, School of Electronic Science and Engineering, Xi'an Jiaotong University, Xi'an 710049, China

${ }^{2}$ State Key Laboratory of Electrical Insulation and Power Equipment, Xi'an Jiaotong University, Xi'an 710049, China

${ }^{3}$ International Research Centre for Renewable Energy, State Key Laboratory of Multiphase Flow in Power Engineering, Xi'an Jiaotong University, Xi'an 710049, China

* Corresponding author (email: zhoudi1220@gmail.com)
} 
structure consisting of electrode-electrolyte-electrode. In order to obtain SCs with high energy density and high power density, besides optimizing electrode materials, improving ionic conductivity and decomposition voltage of the electrolyte are also effective ways. Based on the electrode characteristics, the traditional non-all-in-one sandwich structure FSCs with hydrogel electrolyte as the substrate structure can be divided into two categories, bendable SC and stretchable SC. The electrodes of bendable SCs include the intrinsic bending electrode (such as carbon nanotubes (CNTs), carbon nanofiber (CNF), reduced GO (RGO), graphite paper, graphene, MXene films, or other complexes) and the non-intrinsic bending electrode (such as transition metal oxide/hydroxide, conductive polymer supported on flexible substrates). For stretchable SC, two aspects are mainly studied $[10,12,13]$. (i) The design of electrode or device structure: the non-stretchable electrode material designed into honeycomb-like [14], triangular cone [15], wavy [16], serpentine-shaped [17], and ring spring [18] to achieve stretchability. (ii) Combining electrode materials with elastic or stretchable matrix to obtain conductivity and stretchability, such as polydimethylsiloxane (PDMS) [19], polyurethane (PU) [20], polyurethane acrylate (PUA) [21], acrylate rubber (ACM) [10], textile [6,22], and metal mesh [23]. However, elastic substrate materials are generally non-conductive, while as current collector of stretchable SC, it is necessary to have good electron transfer ability. Unfortunately, the stretchable electrode blended with elastomer always shows low conductivity, which significantly reduces the specific capacitance of the electrode materials and the charge-discharge performance of the device. It is worth mentioning that different elastomers or stretchable substrates have different hydrophilicity or hydrophobicity, so it is necessary to pay attention to the selection of appropriate solvents in the process of electrochemical deposition of conductive polymers.

In the practical application of wearable intelligent electronic devices, certain stress and strain will inevitably be generated under the external environment. However, the application range of bendable SC is limited to a certain extent and the service life will be shortened due to the non-tensile property. The design of electrode or device structures in advance has inherent drawbacks. First, the manufacturing process is complicated and the reproducibility is poor. Second, once the design is formed, the structure and shape cannot be changed to adapt with human bodies (tissue) or other stretchable electronics. Third, these tensile properties are inadequate and limited by the design direction. Therefore, it is necessary to develop other structures or better overall performance of the FSC. Compared with the traditional sandwich structure FSC, the all-in-one structure design has the following advantages: (i) the problem of multiple interface delamination between the electrode and separator is solved and the interface diffusion resistance dramatically declines. (ii) Cyclic bending and stretching will not cause shedding or slippage in practical application. (iii) All-inone FSCs generally possess robust mechanical properties, stable energy output with high energy density, and more importantly, it is easier to implement the self-healing function without adding additional self-healing substrates for hydrogel-based SCs. (iv) All-in-one FSC devices can be manufactured in a variety of shapes and sizes according to the requirements. Therefore, this review introduces the recent achievements and future perspectives of all-in-one FSC, and provides comprehensive highlights.

\section{PERFORMANCE METRICS OF ALL-IN-ONE FSCs}

The electrochemical performance of SCs are mainly characterized by specific capacitance, energy density and power density, as well as other parameters including equivalent series resistance, self-discharge, and cycle life $[24,25]$. Due to the different fabrication processes of allin-one SCs, the electrochemical performance is usually expressed by different measurement units (gravimetric, areal and volumetric). In general, hydrogel-based all-inone SC is a symmetrical SC (SSC), which usually uses areal performance metrics. The total areal specific capacitance of the cell $\left(C_{\text {cell-s }}\right)$ can be acquired through the galvanostatic charge-discharge (GCD) and cyclic voltammetry (CV) curves; especially the GCD test is more common and accurate. It can be calculated by applying Equations $(1,2)$ :

$C_{\text {cell-s }}=\frac{I \cdot \Delta t}{S \cdot \Delta V}$,

$C_{\text {cell-s }}=\frac{\int_{V-}^{V_{+}} I(V) \mathrm{d} V}{S \cdot \Delta V \cdot v}$,

where $I$ is the discharge current during GCD (A); $\Delta t$ is the discharge time during GCD (s); $\Delta V$ is the operating potential range $\left(\Delta V=V_{+}-V_{-}\right)$excluding the $I R \operatorname{drop}(\mathrm{V}) ; S$ is the area of electrode $\left(\mathrm{m}^{2}\right) ; v$ is the scan rate of the CV $\left(\mathrm{V} \mathrm{s}^{-1}\right) ; I$ is the current below the $x$ axis during $\mathrm{CV}(\mathrm{A})$. The areal energy density $\left(E_{\mathrm{s}}, \mathrm{W} \mathrm{h} \mathrm{m}^{-2}\right)$ of the cell can be calculated by Equation (3): 
$E_{\mathrm{s}}=\frac{1}{2} C_{\text {cell-s }} \cdot \Delta V^{2}$.

In fact, the volumetric energy density $\left(E_{\mathrm{V}}, \mathrm{W} \mathrm{h} \mathrm{m}^{-3}\right)$ is more reasonable than the areal energy density and gravimetric energy density $\left(E_{\mathrm{m}}\right)$ in FSCs. The volumetric specific capacitance $\left(C_{\text {cell-v }}\right)$ and $E_{\mathrm{v}}$ of the device can be calculated by Equations $(4,5)$ :

$C_{\text {cell-V }}=C_{\text {cell-s }} \cdot \frac{S}{V_{\text {cell }}}=\frac{C_{\text {cell-s }}}{d}$,

$E_{\mathrm{V}}=\frac{1}{2} C_{\mathrm{cell}-\mathrm{V}} \cdot \Delta V^{2}$,

where $V_{\text {cell }}$ is the volume of the device; $d$ is the thickness of the whole device including electrolyte hydrogel, current collector and packaging $(\mathrm{m})$. Therefore, the thickness of hydrogel is crucial to the volumetric energy density of the device during the experimental design. The maximum volumetric power density $\left(P_{\max -\mathrm{V}}\right)$ of the device can be calculated according to Equation (6):

$P_{\max -\mathrm{V}}=\frac{E_{\mathrm{V}}}{\Delta t}=\frac{1}{4} \frac{\Delta V^{2}}{R_{\mathrm{ESR}} \cdot V_{\text {cell }}}$.

The equivalent series resistance $\left(R_{\mathrm{ESR}}\right)$ can be determined by linear fitting of the $I R$ drop at different current densities from the GCD curves, according to Equation (7) [26]:

$I R_{\text {drop }}=a+b I$,

where $a$ is the voltage difference between the real voltage and the applied voltage of the device; $b$ is twice the $R_{\mathrm{ESR}} ; I$ is the discharge current density. Alternatively, the $R_{\mathrm{ESR}}$ can also be obtained by electrochemical impedance spectroscopy (EIS) in Nyquist plot. It can be calculated by applying Equation (8) [27]:

$R_{\mathrm{ESR}}=R_{\mathrm{s}}+R_{\mathrm{ct}}$,

where $R_{\mathrm{s}}$ is the internal resistance including the inherent resistance of the active material, the ohmic resistance of the electrolyte and the current collector, and the contact resistance between the active material and the current collector; the point of intersection with the real axis at high frequencies in the Nyquist plot represents $R_{\mathrm{s}} ; R_{\mathrm{ct}}$ is the charge transfer resistance at the electrode/electrolyte interface, the diameter of the semicircle at high frequencies in the Nyquist plot represents $R_{\mathrm{ct}}$.

At present, there is no established quantified standard for the flexible evaluation of SCs. Typically, tensile strength, bending, residual strain and compressibility are tested. In view of the one-dimensional (1D) fibrous FSCs, weavability and softness are also very important parameters. One way to test the foldability/compressibility/ stretchability is to statically characterize the SC under different deformation, which only shows that the SC has the corresponding flexibility. However, it is more practical to test the entire device after repeated deformation, which can specifically explain the relationship among mechanical strength, reversibility and electrochemical performance. The tensile properties are tested by placing current collector (carbon paper, sheet metal) on both sides of the pre-stretched hydrogel (electrode included) to form a wavy structure.

\section{HYDROGEL-BASED 2D ALL-IN-ONE SCs}

The ionic conductivity of polymer gel $\left(10^{-4}-10^{-1} \mathrm{~S} \mathrm{~cm}^{-1}\right)$ is much higher than that of dry solid-polymer electrolyte $\left(10^{-8}-10^{-7} \mathrm{~S} \mathrm{~cm}^{-1}\right)$ under ambient conditions, and could reach the same order of magnitude as that of aquaous solution (e.g., $\mathrm{H}_{2} \mathrm{SO}_{4}$ solution: $10^{-1} \mathrm{~S} \mathrm{~cm}^{-1}$ ) $[28,29]$. It has attracted much attention as an electrolyte for FSCs. In general, single-component physical crosslinked polymer, dual-component or multi-component covalently crosslinked polymer are common hydrogel electrolyte substrates due to their excellent hydrophilicity, ionic conductivity and mechanical properties. Typically, polyvinyl alcohol (PVA), polyacrylamide (PAM), and polyacrylic acid (PAA) are used as polymer hydrogel skeleton networks, and the mechanical strength and self-healing of hydrogel are closely related to the whole device. Herein, we provide a summary of hydrogel electrodes or hydrogel electrolytes and their mechanical features, as shown in Table 1.

\section{In-situ polymerization on hydrogel for all-in-one SCs}

These hydrogel-based all-in-one FSC devices are achieved by electrochemically growing active polymers on the surface/sub-surface of the gel electrolyte by in-situ polymerization. Such as polyaniline (PANI) [41,42]. In this strategy, the electrode is embedded in the electrolyte surface layer of the 3D network, which can be effortless to achieve the contact and synchronous deformation of the electrode and electrolyte at molecular level.

\section{Stretchable polyelectrolyte}

PVA hydrogel is a polymer skeleton system with a large amount of water as the medium. It could maintain a certain shape with intrinsic characteristics [43], such as nontoxicity, excellent mechanical properties, and good biocompatibility. Physical crosslinking (freezing/thawing) and chemical/covalent crosslinking (cross-linker) are the most common crosslinking methods. Generally, hydrogels prepared only by mixing PVA with protonic base/ protonic acid/neutral salt, such as $\mathrm{KOH}, \mathrm{H}_{2} \mathrm{SO}_{4}, \mathrm{H}_{3} \mathrm{PO}_{4}$, 
Table 1 Summary of tensile properties and ionic conductivities of hydrogel electrodes, electrolytes and thin films

\begin{tabular}{|c|c|c|c|c|c|}
\hline Substrate & Hydrogel/film & Tensile strain (\%) & Ionic conductivity $\left(\mathrm{mS} \mathrm{cm}^{-1}\right)$ & Self-healing & Reference \\
\hline \multirow[t]{5}{*}{ PVA } & PVA/CNM/PANI electrode & 365 & - & - & {$[30]$} \\
\hline & PVA-KOH electrolyte & - & 30.5 & - & {$[31]$} \\
\hline & DMAOP-PVA/GO-KOH electrolyte & - & 108.7 & - & {$[31]$} \\
\hline & PVA- $\mathrm{H}_{2} \mathrm{SO}_{4}$ electrolyte & 380 & 136.4 & Yes & {$[32]$} \\
\hline & PVA-PMAA- $\mathrm{H}_{3} \mathrm{PO}_{4}$ electrolyte & 475 & 8.5 & - & [33] \\
\hline \multirow[t]{2}{*}{ PAM } & PAM-Au-CNT/PPy electrode & 2380 & - & Yes & {$[34]$} \\
\hline & Agar/HPAAm DN electrolyte & 3400 & 20 & - & [35] \\
\hline \multirow[t]{5}{*}{ PAA } & Modified GO-PAA electrolyte & 950 & 71.6 & Yes & {$[36]$} \\
\hline & $\mathrm{PAA} / \mathrm{Ca}^{2+}$ hydrogel & 1100 & 126 & - & [37] \\
\hline & PAA-PANI-phytic acid film & 500 & 120 & Yes & {$[38]$} \\
\hline & VSNPs-PAA electrolyte & 3700 & - & Yes & [39] \\
\hline & PAA/GO/Fe ${ }^{3+}$ hydrogel & 1186 & - & Yes & {$[40]$} \\
\hline
\end{tabular}

$\mathrm{LiClO}_{4}$ and $\mathrm{LiCl}$, have poor mechanical properties. Yin et al. [44] reported the preparation strategy of an all-in-one integrated SC based on polypyrrole (PPy)-PVA hydrogelpolypyrrole (PHP) with high flexibility. First, physically crosslinked PVA was prepared by three freezing/thawing cycles with each cycle for $12 \mathrm{~h}$. Subsequently, PPy was gradually infiltrated into the hydrogel with the polymerization triggered by an initiator at ice-bath. The assynthesized PHP film exhibited an ionic conductivity of $0.046 \mathrm{~S} \mathrm{~cm}^{-1}$ and the capacitance remained $90 \%$ when the tensile strain reached $110 \%$. The cycling stability of the PHP device demonstrated excellent reliability (a capacitance retention of $97 \%$ after 10,000 cycles at a current density of $1 \mathrm{~A} \mathrm{~cm}^{-2}$ ). As far as we know, this cycling stability is superior to those of previous reports on all-inone FSCs [29]. However, the test details were not reported. In order to give hydrogels stronger mechanical strength and toughness, double-network hydrogels [45], semi-interpenetrating (IPN) hydrogels [46], hydrophobically associating hydrogels [47], and slide-ring hydrogels [48] have also been reported.

In addition to non-covalently crosslinking, covalent crosslinking methods have also been developed to optimize characteristics for appropriate electrolyte materials. Chemical crosslinking can precisely control the crosslinking process and spatial precision of hydrogel by the amount of crosslinking agent, and get stable hydrogel network. For example, Wang et al. [29] proposed the PVA and glutaraldehyde (GA) dual-component chemical hydrogel film $(\mathrm{PCH})$ prepared by the reaction between hydroxyl groups $(-\mathrm{OH})$ and aldehyde groups $(-\mathrm{CHO})$ with the acid as a catalyst, as shown in Fig. 1a. The thickness of the electrode layer (PANI layer) in the PCH film ( Fig. 1b) was controlled by the concentration of the precursor (aniline), but beyond that, controlled polymerization time was also reported [44], with the thickness of the PCH film controlled by the amount of mixed solution and the size of the mold. The PCH film has a high bulk ionic conductivity $\left(0.082 \mathrm{~S} \mathrm{~cm}^{-1}\right)$ and robust tensile properties $(290 \%)$. As a contrast experiment, the PVA film showed quite poor mechanical properties and the surface of the composite film with PANI deposition did not conduct well after drying. This phenomenon is attributed to the fact that the PVA membrane is soluble in aqueous solution and the PANI is encapsulated in the PVA hydrogel. It is well known that the design of a highperformance SC requires high specific capacitance and cyclic stability. This novel configuration SC possesses a large energy density $\left(42 \mu \mathrm{W} \mathrm{h} \mathrm{cm}{ }^{-2}\right)$, cyclic stability $(99 \%$ areal capacitance after 7000 cycles) and flexibility. However, the rate capacity is unsatisfactory when the current density is increased from 0.2 to $2 \mathrm{~mA} \mathrm{~cm}{ }^{-2}$, retaining $70 \%$ of the original areal specific capacitance $\left(488 \mathrm{mF} \mathrm{cm}^{-2}\right)$.

To further explore how to optimize the rate capacity of SCs, based on the above-mentioned research of Wang et al. [29], Bai et al. [49] further treated the chemical crosslinking hydrogel with GA-modified PVA by freezing-casting (named f-PVA), as illustrated in Fig. 1c. This lyophilized hydrogel membrane has stronger van de Waals force and hydrogen-bond interaction between molecular chains than the one without lyophilization, which not only shows high ion migration rate, robust mechanical strength, but also has excellent water retention ability. The hydrogel appears opaque milky white after freezing due to the formation of crystalline regions (Fig. 1d: (d2)). The results reveal that the conductivity of 
a
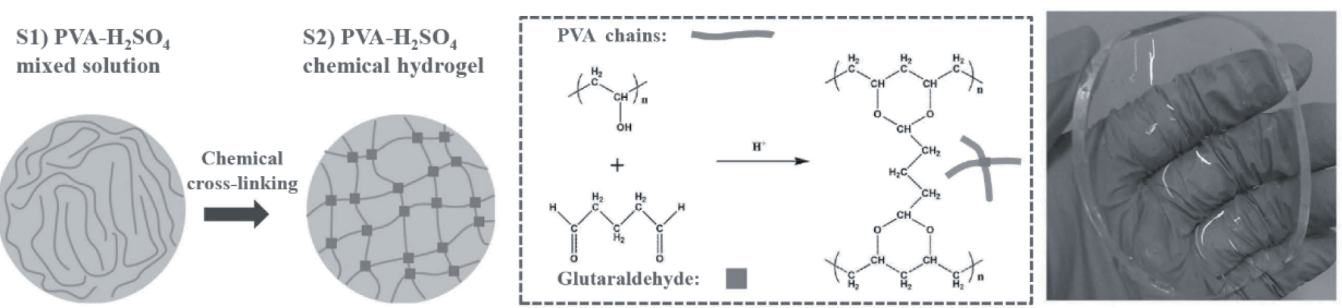

b
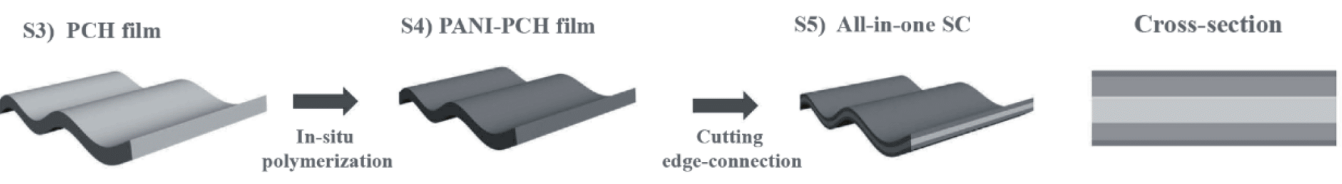

C
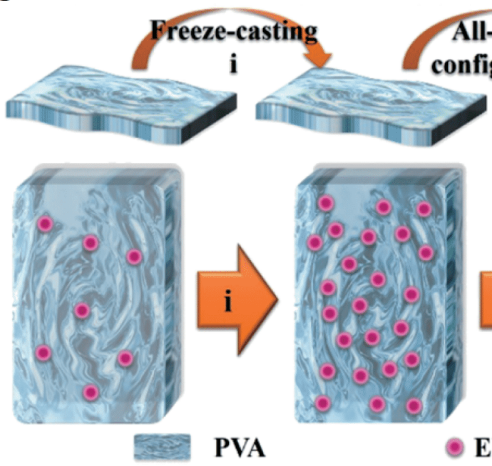

All-in-one
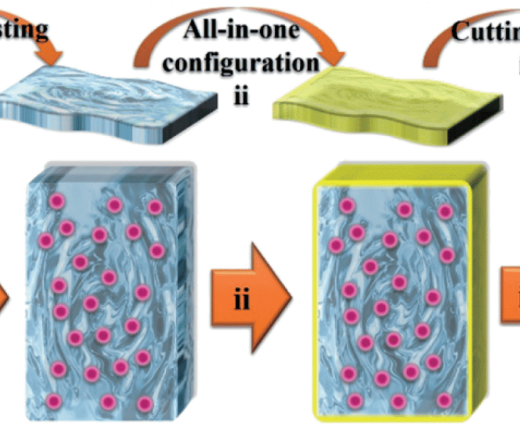
ii tting edges
iii
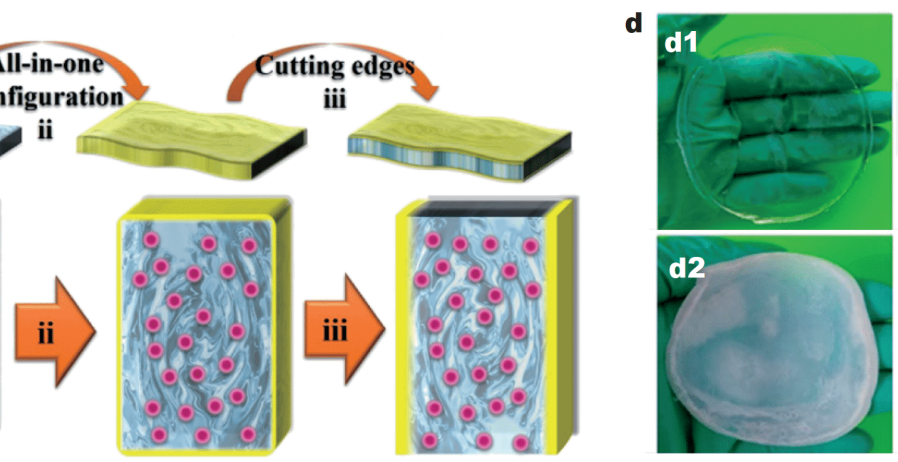

PVA

- Electrolyte ions

PPy
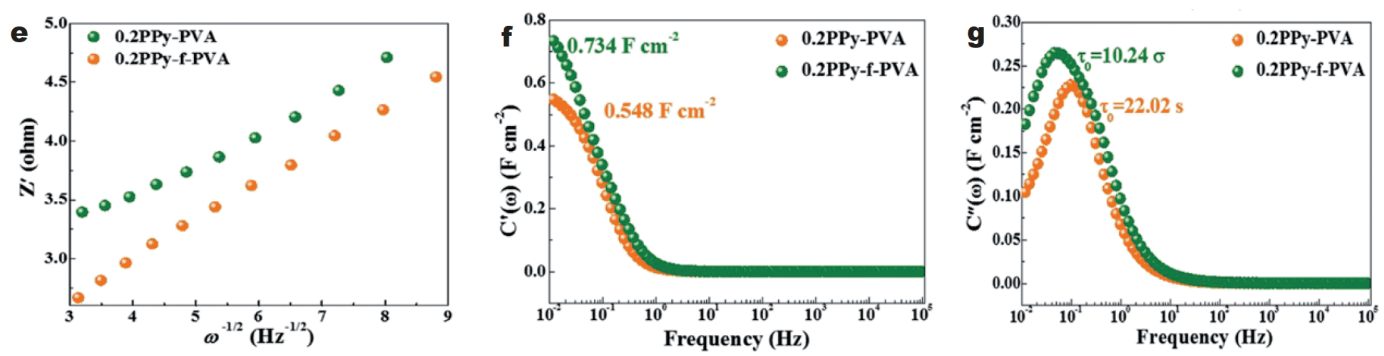

Figure 1 (a) Schematic illustration of PVA covalent crosslinking and PCH film. (b) Schematic illustration of the preparation of all-in-one SC. Reproduced with permission from Ref. [29]. Copyright 2015, Wiley-VCH. (c) The freezing-casting PVA (f-PVA) fabrication route and mechanism for introducing ions. (d) The digital images of PVA prepared via freezing-casting (d2) and without freezing-casting (d1). (e) Relationship between $Z$ and $\omega^{-1 / 2}$. Real (f) and imaginary (g) parts of the complex capacitance dependent on frequency. Reproduced with permission from Ref. [49]. Copyright 2019, American Chemical Society.

f-PVA $\left(0.79 \mathrm{~S} \mathrm{~cm}^{-1}\right)$ is greater than that of PVA $\left(0.09 \mathrm{~S} \mathrm{~cm}^{-1}\right)$ without freezing, which is also the main factor to improve the rate performance. Specifically, when the current density is increased from 1 to $50 \mathrm{~mA} \mathrm{~cm}^{-2}$, the areal specific capacitance retains $58.2 \%$ of the original capacitance $\left(644.4 \mathrm{mF} \mathrm{cm}^{-2}\right.$ ).

The viewpoint was further proved by electrochemical properties. Fig. 1e shows the relationship between the real part of the impedance $\left(Z^{\prime}\right)$ in the low-frequency region and $\omega^{-1 / 2}$, which can be expressed as Equation (9):
$Z^{\prime}=a+b \omega^{-1 / 2}$

where $b$ is the diffusion impedance, reflecting the characteristic of the diffusion channel in the internal structure of the material. $\omega$ is the angular frequency, which is equal to $2 \pi f$. According to Fig. 1e, 0.2PPy-f-PVA presented a lower slope than $0.2 \mathrm{PPy}-\mathrm{PVA}$ at the low-frequency region, which indicated good ion diffusion kinetics and excellent rate performance. The function of capacitance to frequency of SC is often used to characterize the rate capacitance of the device, it can be described as 
Equation (10):

$$
\begin{aligned}
C(\omega) & =\frac{-Z^{\prime \prime}(\omega)}{\omega|Z(\omega)|^{2}}-j \frac{Z^{\prime}(\omega)}{\omega|Z(\omega)|^{2}} \\
& =C^{\prime}(\omega)-j C^{\prime \prime}(\omega),
\end{aligned}
$$

where $C^{\prime}(\omega)$ is the real part of the complex capacitance, and the value of $C^{\prime}$ in the low-frequency range is close to the specific capacitance value measured in galvanostatic charge-discharge (GCD). Fig. If shows $C^{\prime}(\omega)$ of $0.2 \mathrm{PPy}-\mathrm{f}-$ PVA at $0.01 \mathrm{~Hz}\left(734 \mathrm{mF} \mathrm{cm}^{-2}\right)$ is approximate to the capacitance value calculated by the GCD $\left(644.4 \mathrm{mF} \mathrm{cm}^{-2}\right)$. Here $C^{\prime \prime}(\omega)$ is the imaginary part of the complex capacitance, which is related to the irreversible energy dissipation of the SC. The maximum value of $C^{\prime \prime}$ corresponds to the characteristic frequency $f_{0}\left(f_{0}=1 / \tau_{0}\right)$, and the relaxation time constant $\left(\tau_{0}\right)$ is defined as the minimum time required for the device to release all the energy with an efficiency of more than 50\% [50]. Low $\tau_{0}$ indicates fast charge-discharge rate and good rate capacitance, as shown in Fig. 1g.

However, hydrogels prepared by chemical crosslinking can enhance mechanical properties at the expense of selfhealing properties due to the abrupt decline in the formation of hydrogen bonds. It is well known that human body and skin activities are accompanied by a certain degree of stretchability, which inevitably leads to device damage during repeated activities, making self-healing hydrogel film highly desirable to extend the service life of wearable electronic devices.

\section{Self-healing polyelectrolyte}

Up to now, many literatures have reported that selfhealing SCs are realized by adding additional self-healing materials to package the electrode or integrated SCs (e.g., carboxylated polyurethane) $[18,51,52]$. The self-healing performance of this method is mainly determined by the additional self-healing material, which has little relation to the energy storage device itself, resulting in the low energy density of the whole device. In fact, the intrinsic self-healing of materials mainly depends on reversible covalent bond (such as cycloaddition reaction, reversible acylhydrazone bonds, disulfide bonds, boron-oxygen bonds and reversible free radical reaction) and dynamically reversible non-covalent interaction (such as hydrogen bonds, ionic bonds, metal-ligand interaction, $\pi-\pi$ stacking, hydrophobically associating and host-guest interaction) [53].

In order to optimize and improve the structural integrity and mechanical properties of PVA hydrogels. Guo et al. [32] presented the fabrication of the healable all-in- one FSC configuration by in-situ polymerization of PANI and single wall carbon nanotubes (PANI-SWCNTs) onto the physically crosslinked PVA- $\mathrm{H}_{2} \mathrm{SO}_{4}$ hydrogel film with freezing/thawing treatment. The crystallized regions formed during the freezing process as cross-linking points connected the random disordered PVA molecular chains to form a 3D network structure. The physically crosslinked hydrogel electrolyte film presented an ionic conductivity of $0.136 \mathrm{~S} \mathrm{~cm}^{-1}$ and could be stretched to $380 \%$ of its original length. However, the PVA hydrogel cured at room temperature without freezing did not form a membrane over the same time period. Besides, soaked in aqueous solution for $48 \mathrm{~h}$, the hydrogel film with freezing/thawing treatment only resulted in swelling and negligible dissolvation, while the one without freezing/ thawing treatment (longer film formation time) was almost completely dissolved, consistent with the aforementioned research of Wang et al. [29], indicating that the preparation via freezing/thawing treatment can dramatically improve the stretchability properties of the PVA hydrogels. The specific capacitance of the self-healing SC can reach $15.8 \mathrm{mF} \mathrm{cm}^{-2}$ at the current density of $0.044 \mathrm{~mA} \mathrm{~cm}^{-2}$. After five times of self-healing cycles, the specific capacitance value can still retain about $80 \%$. In summary, the PVA hydrogels with merely a blended solution (such as, PVA- $\mathrm{H}^{+} / \mathrm{OH}^{-} / \mathrm{Na}^{+}$) have been widely used in the field of FSCs. However, as an electrolyte it is not suitable for the preparation of all-in-one integrated SCs as a structural substrate for deposition of electrochemical active materials. This is the reason why the preparation of PVA hydrogels requires chemical crosslinking agents, freezing/thawing processes or other processing methods.

As the strength and energy consumption of singularcrosslinked interactions is inferior to that of dual-crosslinked hydrogels, such as the crosslinking between dual hydrogen bonds, hydrogen bonds and metal ion coordination bonds confer better mechanical properties and self-healing properties than weak singular-crosslinked hydrogels. For example, Yang et al. [54] well designed a SC based on multiple hydrogen bonds crosslinking with excellent mechanical properties, fatigue-resistant and selfhealing properties. First, PVA was used as a molecular template. Poly(N-hydroxyethyl acrylamide) (PHEA) was polymerized along the PVA chains to form ordered hydrogen bonds, so as to realize the physical self-crosslinking hydrogel with multiple hydrogen bonds. Subsequently, during the in-situ polymerization of PANI, phytic acid was added as acid dopant and crosslinking agent to effectively improve the tear resistance of the SC, 


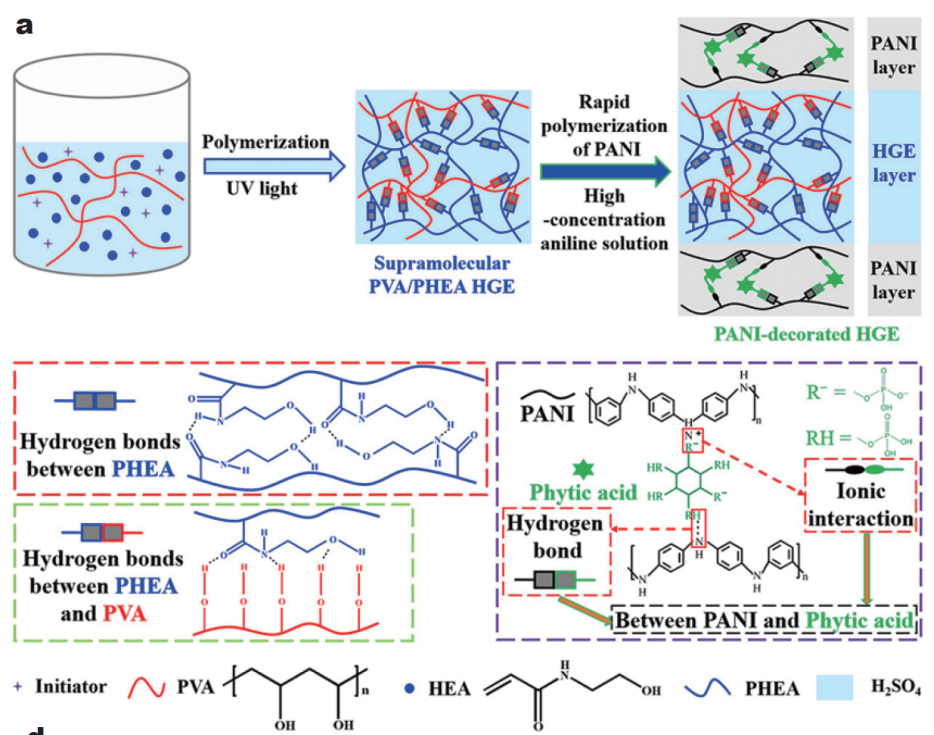

。

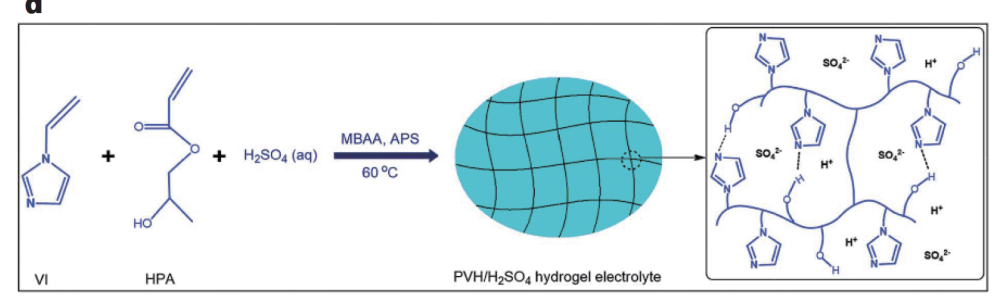

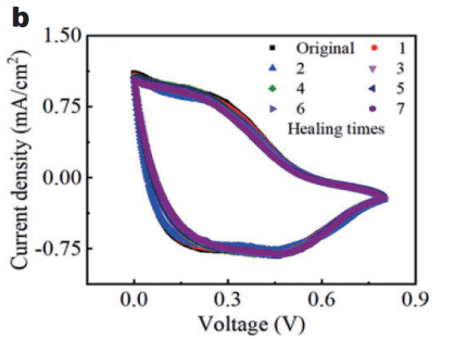
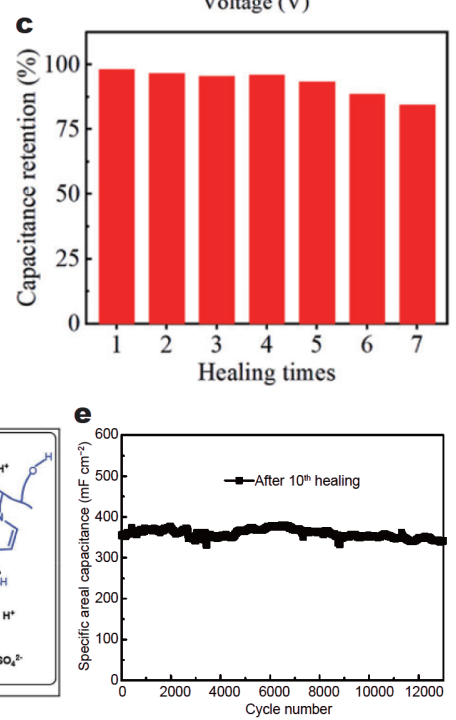

Figure 2 (a) Schematic illustration of the preparation of PANI-PHEA/PVA all-in-one SC. (b) CV curves (scan rate of $5 \mathrm{mV} \mathrm{s}$ ) of the SC after different self-healing times. (c) Residual capacitance of the SC after different self-healing times by GCD (current density of $0.5 \mathrm{~mA} \mathrm{~cm}{ }^{-2}$ ). Reproduced with permission from Ref. [54]. Copyright 2020, American Chemical Society. (d) PVH hydrogel synthesis route. (e) Cyclic stability of the SC after the 10th self-healing. Reproduced with permission from Ref. [55]. Copyright 2018, the Royal Society of Chemistry.

which was attributed to the ionic bonds and hydrogen bonds formed between phytic acid and polyaniline, as shown in Fig. 2a. The multiple hydrogen bonds crosslinking method can effectively solve the time-consuming disadvantage of freezing method. The all-in-one FSC exhibited a high specific capacitance of $98 \mathrm{mF} \mathrm{cm}^{-2}$ at a current density of $0.2 \mathrm{~mA} \mathrm{~cm}^{-2}$, energy density of $8.48 \mu \mathrm{W} \mathrm{h} \mathrm{cm}{ }^{-2}$ and a power density of $78.52 \mu \mathrm{W} \mathrm{cm}{ }^{-2}$. Fig. $2 \mathrm{~b}$ shows the $\mathrm{CV}$ curves (scan rate of $5 \mathrm{mV} \mathrm{s}^{-1}$ ) of different self-healing times. Fig. $2 c$ demonstrates a slight decrease in capacitance retention with the increase of selfhealing times, and after the 7th self-healing cycles, it still retains $84 \%$ of the capacitance.

Further studies focus on overcoming the problem that traditional SCs sacrifice partial capacitance storage with the increase of self-healing times. Liu et al. [55] prepared the self-healing hydrogel (PVH) with vinylimidazole (VI) and hydroxypropyl acrylate (HPA) covalently crosslinked at the molecular level, and then deposited the PANI active material in the same way, as shown in Fig. 2d. Fig. 2e shows that the SC had excellent self-healing and high specific capacitance properties, simultaneously. After cutting/healing for 10 times, it retained a specific capacitance of $341.7 \mathrm{mF} \mathrm{cm}^{-2}$ at $2 \mathrm{~mA} \mathrm{~cm}^{-2}$ after $13,000 \mathrm{cy}-$ cles, exhibiting a retention rate of $96 \%$. This may be related to the interaction between PANI molecular chain and imidazole ring ( $\pi-\pi$ and $\pi$-cation interactions), which inhibits the pulverization of PANI molecular structure in the long-term cycling process.

\section{Compressible polyelectrolyte}

The dual inherent compressibility and stretchability are crucial for flexible electronic devices to withstand additional stresses in practical applications. Among the numerous polymer hydrogels, the widely used PVA-based, polyethylene terephthalate (PET) and polyimide (PI) gel electrolytes have no compressibility. In contrast, PAMand PAA-based gel electrolytes are proved to be compressible [56]. Liao et al. [57] reported that dual-crosslinked PAA-based hydrogel (hydrophobic associating and 
hydrogen bond) exhibited considerable compressibility and self-healing. Similarly, compressible all-in-one SCs have also been reported. For example, Hu et al. [58] reported that PAA hydrogel film was prepared with ammonium persulphate (APS) as initiator, and then soaked in a solution containing CNTs and pyrrole (Py) monomer to obtain a quasi-solid-state all-in-one FSC by in-situ polymerization. In this preparation process, the role of CNTs is primarily to provide perfect conductive pathways, while PPy is to form electrochemical redox reactive sites and to facilitate the surface deposition of CNTs on the electrolyte. The results show that the electrochemical performance of the SC remains in the aboriginality when the SC is compressed by $60 \%$, and the effect of compressed electrolyte on electrochemical performance is negligible and may even be slightly beneficial [59]. However, the electrochemical performance of all-in-one devices generally decreases significantly with the increase of tensile strain. The published literatures have also reported that the electrochemical performance of traditional sandwich structure SC with wrinkled electrodes and gel electrolyte has slightly increased under tensile strain [35]. It is worth noting that CNTs cannot be embedded into the electrolyte at a large scale without Py. Interestingly, Wang et al. [10] suggested that multiwalled CNTs (MWCNTs) could promote electrochemical deposition of conductive polymers on acrylate rubber (ACM) substrates. Hence, we suspect there is great possibility synergistic effect between the conducting polymer (monomer) and the CNTs to mutually reinforce the deposition on the polymer surface/sub-surface.

\section{Hydrogel electrode and hydrogel electrolyte recombination for all-in-one SCs}

All-in-one FSC could be prepared by recombination of hydrogel electrode and hydrogel electrolyte, which is different from the traditional sandwich structure by nonhydrogel electrode due to the strong interface adhesion without obvious delamination phenomenon and low interface resistance. Conductive polymer hydrogel electrode and hydrogel electrolyte recombination can achieve ion transport channels at molecular level, which has particular prospect in flexible wearable electronic devices. Some typical hydrogel electrodes are prepared by incorporating the host hydrogel matrix like PANI [60], PEDOT-PSS [33] and PPy [61] with conducting polymers. Hydrogel electrolyte and hydrogel electrode constitutes sandwich structure through pressure condition integration into the all-in-one SCs. A large amount of hydrogen bonding or other forms of force (such as co- ordination bond) between the gels form strong self-adhesion, so there is no obvious delamination at the interface with low interfacial resistance and remarkable mechanical properties with reversible deformation under external forces.

For example, Zeng et al. [56] prepared a PAM/SA (sodium alginate) dual-network crosslinked hydrogel electrolyte and electrode, respectively, as shown in Fig. 3a. It is worth mentioning that the tensile strain of hydrogel electrolyte can reach $2400 \%$, and the hydrogel electrode also can exceed $1000 \%$, which can achieve arbitrary deformation. As a functional component, SA with long chain structure is randomly distributed in the PAM crosslinked skeleton, and the excellent mechanical properties are attributed to the interaction of macromolecular entanglement and enough hydrogen bonds. The fabricated PEDOT:PSS/CNT based symmetrical SC (SSC) with $\mathrm{K}_{3}\left[\mathrm{Fe}(\mathrm{CN})_{6}\right] / \mathrm{K}_{4}\left[\mathrm{Fe}(\mathrm{CN})_{6}\right]$-PAM-SA gel electrolyte demonstrates a areal capacitance of $232 \mathrm{mF} \mathrm{cm}^{-2}$ at a scan rate of $5 \mathrm{mV} \mathrm{s}^{-1}$, energy density of $3.6 \mu \mathrm{W} \mathrm{h} \mathrm{cm}{ }^{-2}$ $\left(24 \mu \mathrm{W} \mathrm{h} \mathrm{cm}{ }^{-3}\right)$ at the power density of $0.2 \mathrm{~mW} \mathrm{~cm}^{-2}$ $\left(1.33 \mathrm{~mW} \mathrm{~cm}^{-3}\right)$, which are far higher than that of the SSC without $\mathrm{K}_{3} \mathrm{Fe}(\mathrm{CN})_{6} / \mathrm{K}_{4} \mathrm{Fe}(\mathrm{CN})_{6}$ redox couple and merely PEDOT:PSS or CNT based SSC. The main reason is that the redox couple provides a certain pseudocapacitance (Fig. 3b). This redox process can be described by Equation (11):

$\mathrm{Fe}(\mathrm{CN})_{6}^{4-} \rightleftarrows \mathrm{Fe}(\mathrm{CN})_{6}^{3-}+\mathrm{e}^{-}$.

However, the SSC with redox couple gel electrolyte reveals unsatisfactory coulomb efficiency due to the enhanced self-discharge effect. Many studies demonstrate that the introduction of redox additives in gel electrolytes can effectively improve the energy density, power density and ion conductivity, such as ethyl viologen (EV) dibromide [62], sodium iodide (NaI) [63], $\mathrm{K}_{3}\left[\mathrm{Fe}(\mathrm{CN})_{6}\right]$ [64], hydroquinone (HQ) $[65,66]$, ammonium molybdate $\left[\left(\mathrm{NH}_{4}\right)_{2} \mathrm{MoO}_{4}\right][67,68]$, nickel nitrate $\mathrm{Ni}\left(\mathrm{NO}_{3}\right)_{2}$ [69], and decamethylcobaltocene (DmCc) [70].

Besides these studies on hydrogen bonding between electrode and electrolyte, many studies focus on multiple interfacial interactions. Wang et al. [60] reported that the tensile strength, tensile reversibility, and self-healing of PAA-based electrolyte with $\mathrm{Co}_{2}{ }^{+}$were superior to that of the one without $\mathrm{Co}_{2}^{+}$due to the dual interaction between the metal coordination bonds $\left(\mathrm{Co}_{2}{ }^{+}\right)$and the hydrogen bonds. Likewise, the synergistic effect of hydrogen bonds and metal coordination bonds exists at the interface of electrode and electrolyte. The SC exhibited an equivalent series resistance $\left(R_{\mathrm{ESR}}\right)$ of $3.7 \Omega$, capacitance of $162 \mathrm{~F} \mathrm{~g}^{-1}$ 
a
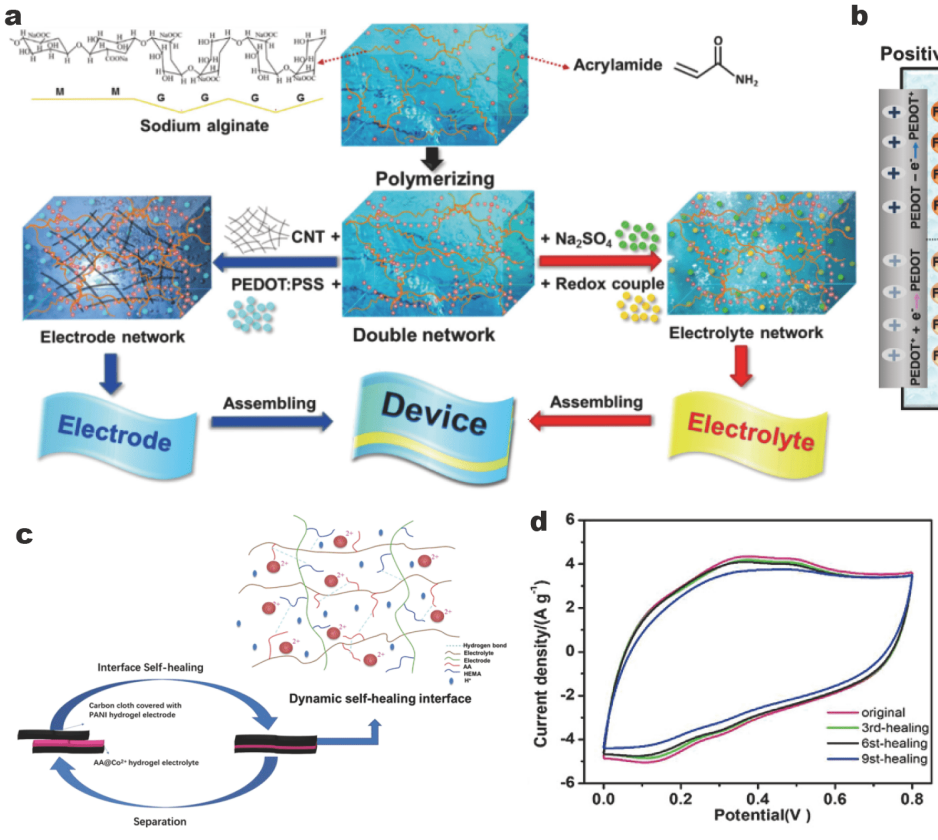
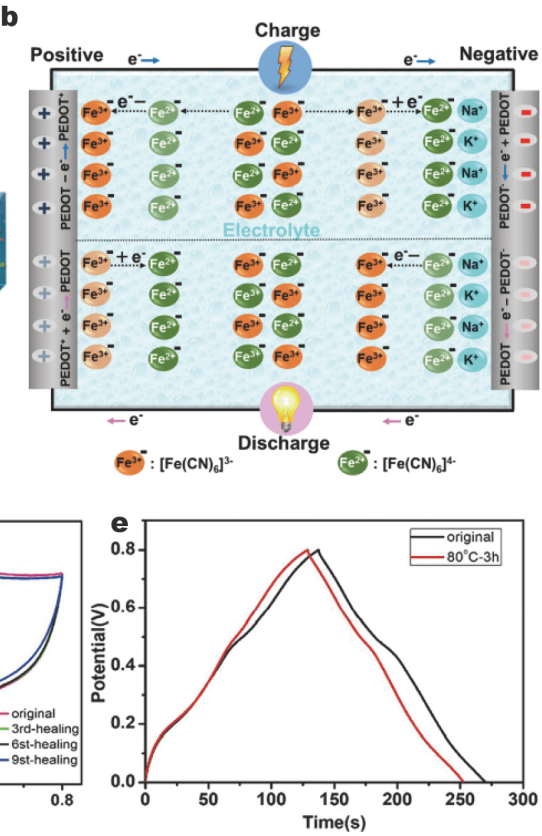

Figure 3 (a) Schematic diagram of the preparation of hydrogel electrode and hydrogel electrolyte. (b) Schematic diagram of the charge storage principle of the SC. Reproduced with permission from Ref. [56]. Copyright 2020, Elsevier. (c) Schematic diagram of interface separation and selfhealing in horizontal direction. (d) CV curves (scan rate of $10 \mathrm{mV} \mathrm{s}^{-1}$ ) of SC after different self-healing times. (e) GCD curves of the electrode recovery (current density of $2 \mathrm{~A} \mathrm{~g}^{-1}$ ). Reproduced with permission from Ref. [60]. Copyright 2020, Academic Press Inc.

at $0.5 \mathrm{~A} \mathrm{~g}^{-1}$, energy density of $14.22 \mathrm{~W} \mathrm{~h} \mathrm{~kg}^{-1}$ at a power density of $200.6 \mathrm{~W} \mathrm{~kg}^{-1}$ for a $0.8 \mathrm{~V}$. Fig. $3 \mathrm{c}$ illustrates the process of separation and self-healing of the electrode and electrolyte in the horizontal direction. The CV curves (Fig. 3d) show that the capacitance was retained by $92 \%$ after nine times of separation/self-healing, indicating a strong interaction through the design of hydrogen bonds and metal coordination bonds at the interface. Even more interestingly, the study also shows that the electrode with economic effects could be reversibly recovered with little effect on electrochemical properties under $80^{\circ} \mathrm{C}$ aqueous solution (Fig. 3e). Hydrogel-based all-in-one SCs can be more selective in flexible electronic devices.

\section{NON-HYDROGEL-BASED 2D ALL-IN-ONE SCs}

Compared with the hydrogel-based all-in-one FSC, the manufacture of flexible substrates (separator) of the nonhydrogel-based all-in-one SCs is simpler and easier to be accurately controlled. They are easier to be assembled in series or parallel for the integration application of the device, but generally do not have the tensile and selfhealing properties. It is stated here that non-hydrogelbased SCs do not have pre-prepared hydrogels as sandwich structure centers but that does not mean they cannot contain hydrogel electrolytes.

\section{Electrodeposition technology}

It is well known that electrodeposition is an effective method for preparing thin films with plasticity, uniform and controllable thickness, smooth surface and close contact with substrate, and could adapt to extreme requirements of substrate. Gao et al. [71] proposed electrodeposition to prepare compact all-in-one asymmetric SC (ASC) with high energy density and flexibility, as shown in Fig. 4a. The porous polyamide nanofiber membrane was prepared by an electrospinning process as the separator, and CNTs were assembled into the 3D film surfaces as current collectors (Fig. 4a). Hydroxyl oxidize iron nanosheets (FeOOH NS) and manganese dioxide nanowires $\left(\mathrm{MnO}_{2} \mathrm{NW}\right)$, as active materials, were electrodeposited on each side of CNT-modified polyamide film as negative electrode and positive electrode, respectively. Fig. $4 \mathrm{~b}$ shows the scanning electron microscopy (SEM) image of the cross section of the all-in-one ASC. The assembled all-in-one ASC achieved a specific capacitance of $70 \mathrm{~F} \mathrm{~g}^{-1}$ at $0.5 \mathrm{~A} \mathrm{~g}^{-1}, 96.5 \%$ capacitance retention and almost $100 \%$ coulomb efficiency at a current density of $2 \mathrm{~A} \mathrm{~g}^{-1}$ (Fig. 4c, d) after 10000 cycles. High specific capacitance makes it possible for flexible wearable intelligent electronic devices.

Similarly, Liu et al. [11] reported sputtering metallic gold on each side of the commercial melamine foam (MF) 

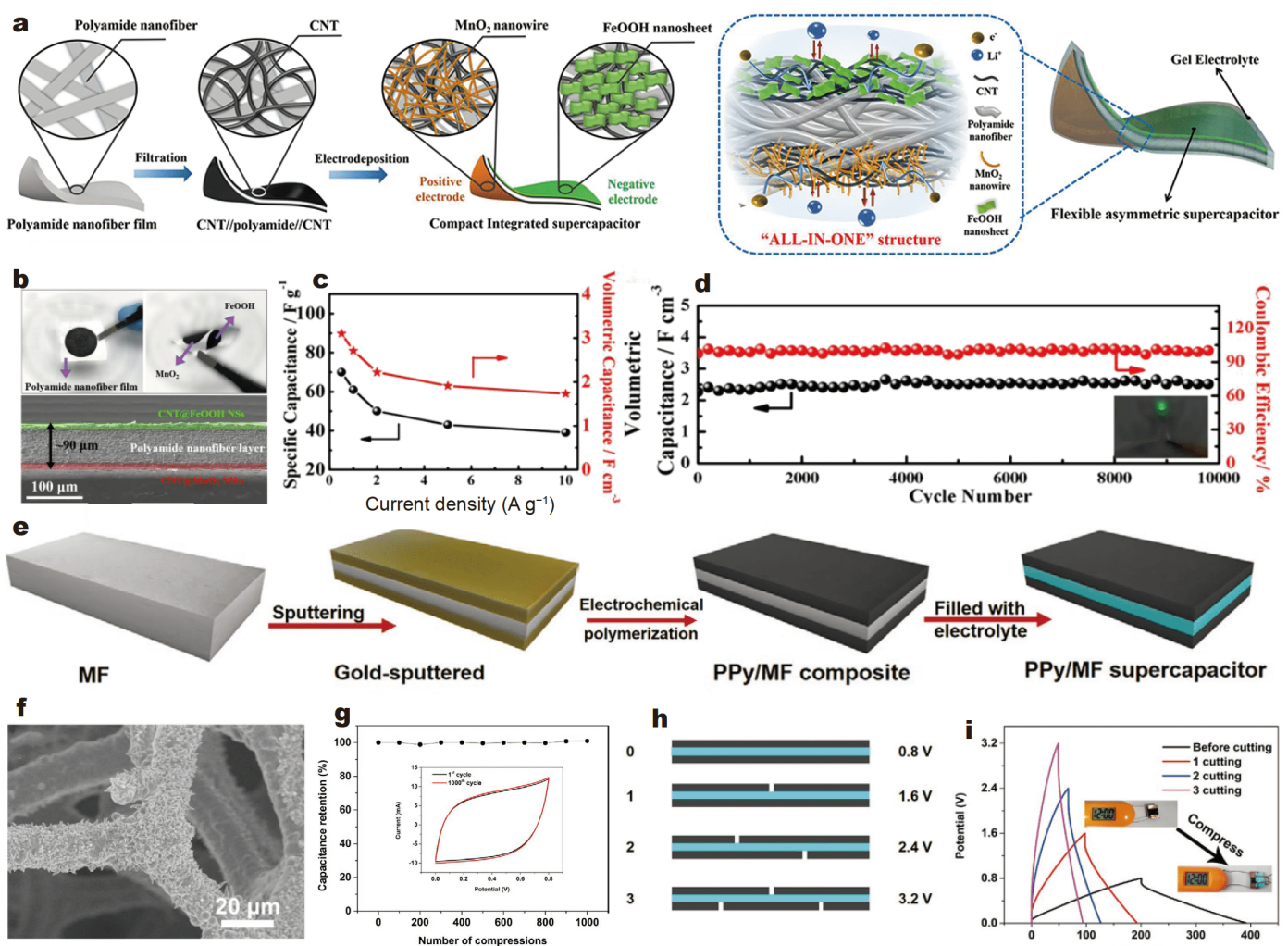

Gold-sputtered

PPy/MF composite

PPy/MF supercapacitor
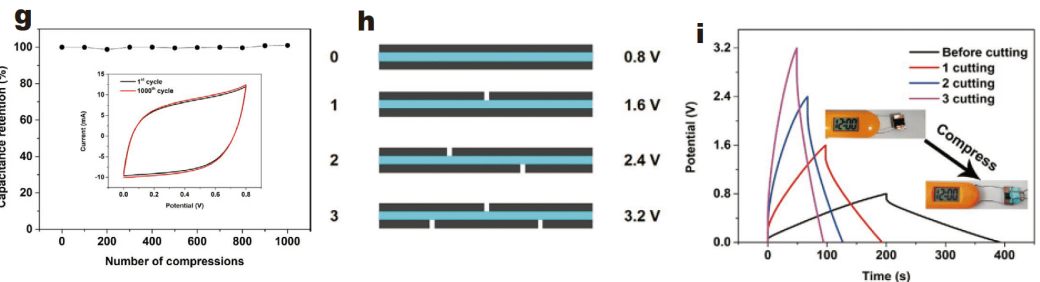

j

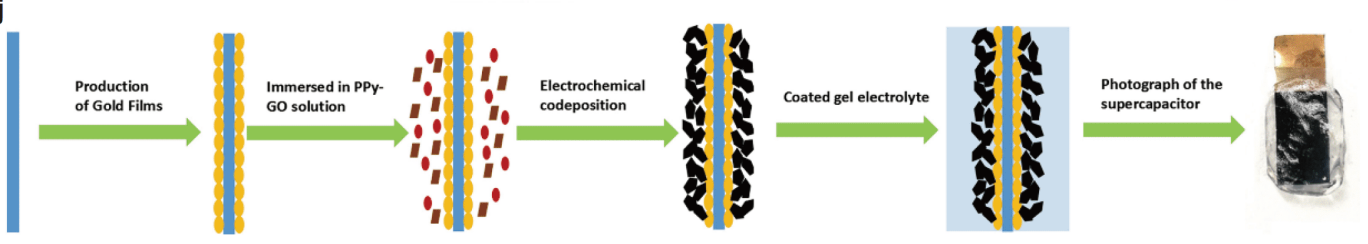

Figure 4 (a) The preparation and structure diagram of the $\mathrm{MnO}_{2} \mathrm{NW} / / \mathrm{FeOOH}$ NS all-in-one SC. (b) Digital photographs and SEM image of the allin-one SC. (c) Rate performance of ASC. (d) Cyclic stability of the ASC (current density of $2 \mathrm{~A} \mathrm{~g}^{-1}$ ). Reproduced with permission from Ref. [71]. Copyright 2018, American Chemical Society. (e) Schematic diagram of the manufacturing process of PPy/MF all-in-one SC. (f) SEM image of PPy/ MF-40. (g) The capacitance retention of the device for 1000 compression cycles at a strain of $50 \%$. (h) The schematic diagram of the cutting method to extend voltage window. (i) The GCD curves under different cutting times and when cutting once before and after the compression to light up the LCD electronic watch. Reproduced with permission from Ref. [11]. Copyright 2019, Wiley-VCH. (j) Schematic diagram of the preparation of PPy-GO// PPF//PPy-GO all-in-one SC. Reproduced with permission from Ref. [72]. Copyright 2018, Elsevier.

as a conductive layer, the electrodeposited PPy as active material for an SSC, and the intermediate pure MF as an insulating layer, as shown in Fig. 4e. In order to obtain an SC with excellent overall performance, it is necessary to consider the deposition time to meet the balance between flexibility, rate performance and specific capacitance. From the SEM image (Fig. 4f) of PPy/MF-40 (deposition time $40 \mathrm{~min}$ ), we can see that the deposited PPy presents a microcone structure with a high specific surface area, and the addition of electroactive sites in the surface is beneficial to the electrochemical performance. The SSC exhibited a high specific capacitance of $2.86 \mathrm{~F} \mathrm{~cm}^{-3}$, an

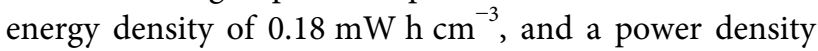

up to $22.97 \mathrm{~mW} \mathrm{~cm}^{-3}$; moreover, the specific capacitance did not change significantly after 1000 compression cycles (Fig. 4g). More delightfully, it can be connected in series by simply cutting itself to extend the voltage window without using extra wires to connect the SCs in series (Fig. 4h). The relevant parameters in the series circuit can be calculated according to Equations $(12,13)$ :

$C_{\text {cell }}=\frac{1}{n} C_{\mathrm{s}}$,

$U_{\text {cell }}=n U_{\mathrm{s}}$,

where $U_{\text {cell }}$ and $C_{\text {cell }}$ are total operating voltage and total specific capacitance, respectively; $U_{\mathrm{s}}$ and $C_{\mathrm{s}}$ are the op- 
erating voltage and specific capacitance of a single capacitor, respectively; $n$ represents the number of identical SCs. Fig. 4i demonstrates the SC can drive the electronic watch before and after compression under once cutting, suggesting the feasibility of this method.

Due to the inherent compact structure of PPy, it is difficult for ions to migrate into the deeper layers of PPy, and high areal specific capacitance usually requires more mass (thicker) of the active materials, which contradicts the high rate performance. To address this problem, Wang et al. [72] reported an all-in-one SC formed by electrodeposition of PPy-graphene oxide (PPy-GO) on a gold-plated polypropylene film (PPF) substrate, as shown in Fig. 4j. GO with a large specific surface area and both hydrophilic and hydrophobic groups can effectively disperse Py monomers, and act as a structural scaffold to promote the formation of 3D porous electrode materials. The results show that the electrochemical performance (areal specific capacitance, rate and cyclic stability) was significantly improved compared with that of PPy-based SC.

\section{Laser direct writing and electrodeposition technologies (MSCs)}

MSC, also known as in-plane SC, is a novel type of ultrathin and ultra-small micro-energy storage devices. It integrates the electrode material, electrolyte and current collector in the same plane, which significantly reduces the thickness of the vertical axis. This planar configuration provides a planar channel for the electrolyte ions while meeting the integration needs. Compared with traditional micro/nano processing techniques such as lithography and mask micropatterning on the substrate, the laser direct writing technique can not only improve the machining precision of the electrode, but also design special patterns arbitrarily, and simultaneously generate multiple energy storage devices in limited space. Meanwhile, previous efforts have been primarily focused on the capacitive carbonaceous material-based planar MSCs [73]. In recent years, pseudocapacitive materials, such as MXene [74-77], conductive metal oxides and polymer composites [78], have also been explored in MSCs. For example, Gao et al. [78] used laser direct writing technology to pattern interdigital electrodes on indium tin oxide-polyethylene terephthalate (ITO-PET) film, and then reduced GO (RGO, current collector), MWCNTs, PPy, and manganese dioxide $\left(\mathrm{MnO}_{2}\right)$ were electrodeposited on each interdigital electrodes to produce PPy@MWCNT//MnO $@$ @Py@MWCNT asymmetric MSCs (AMSCs), as shown in Fig. 5. The large-scale integration strategy of MSCs on the flexible film can adjust the output voltage according to the demand by changing the connection of the output port. Among them, the energy density of a single device can reach $12.16 \mu \mathrm{W} \mathrm{h} \mathrm{cm}{ }^{-2}$ corresponding to a power density of $136.8 \mu \mathrm{W} \mathrm{cm}{ }^{-2}$ at a scan rate of $5 \mathrm{mV} \mathrm{s}^{-1}$ and voltage window of $1.6 \mathrm{~V}$.

\section{Reduction of GO technology}

Graphene is widely used as an electrode of carbon-based materials for SCs, in the preparation of all-in-one SCs. $\mathrm{GO}$ is a typical precursor to prepare RGO by removing surface oxygen-containing functional groups. Laser-induced graphene can also be obtained by one-step laser scribing of polymer precursors such as PI and polysulfone [79]. Du et al. [80] reported that a membrane-like FSC

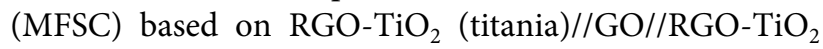
sandwich structure was fabricated by layer-by-layer as-

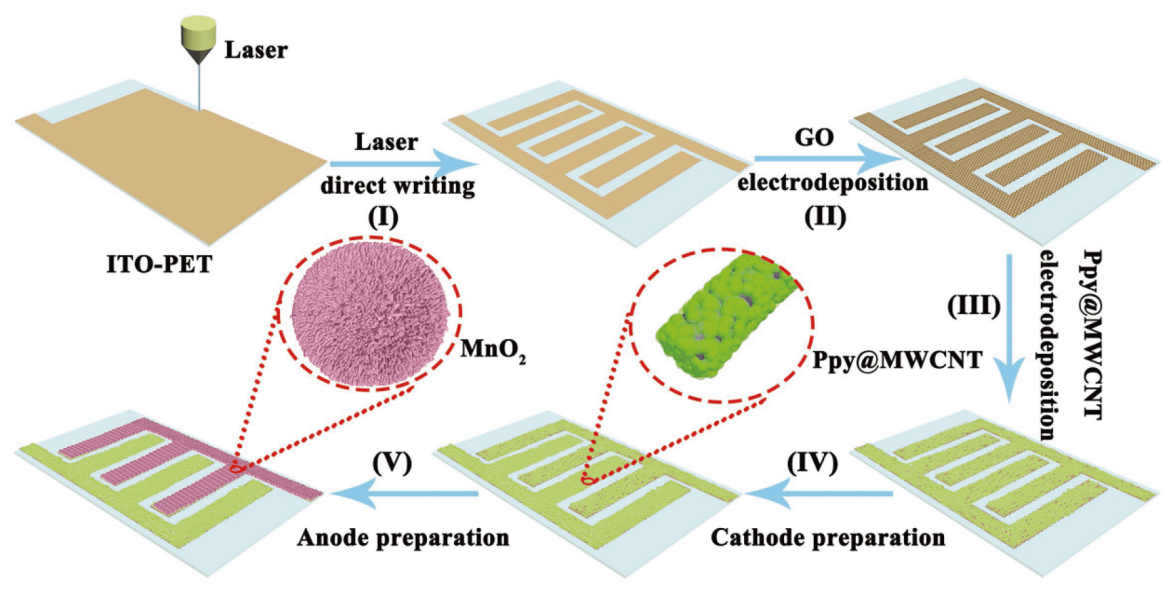

Figure 5 Schematic diagram of the preparation of AMSC. Reproduced with permission from Ref. [78]. Copyright 2018, Wiley-VCH. 


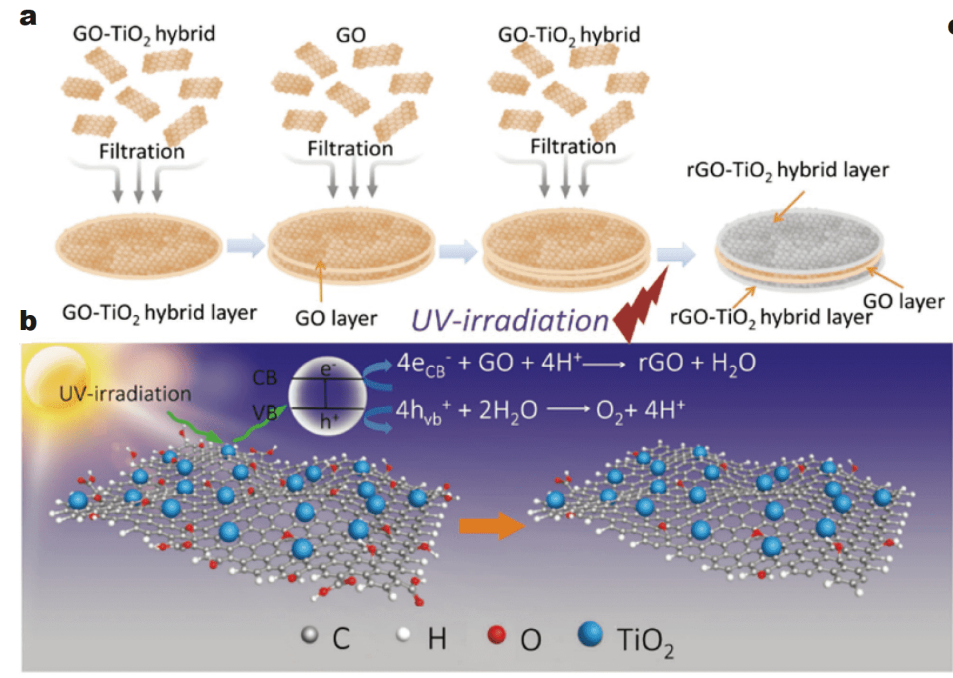

d

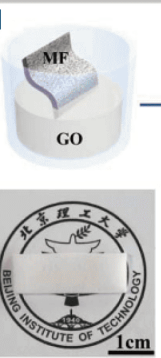

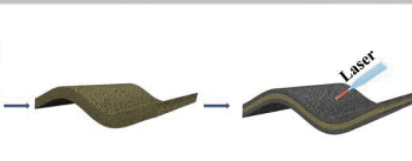
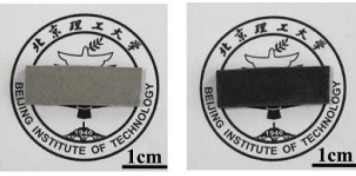

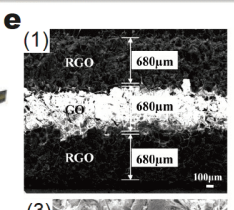

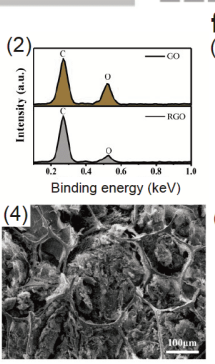

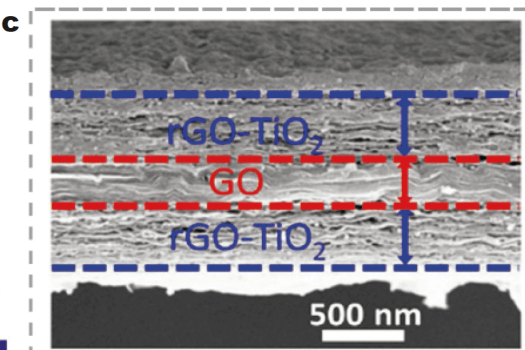

PET
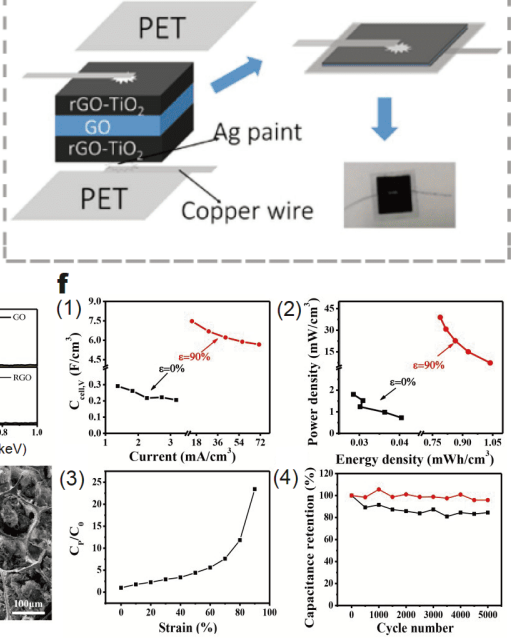

Figure 6 (a) Schematic diagram of the preparation of $\mathrm{RGO}-\mathrm{TiO}_{2} / \mathrm{GO} / \mathrm{RGO}-\mathrm{TiO}_{2}$ hybrid film. (b) Mechanism of GO reduction. (c) Cross-section SEM image of $\mathrm{RGO}-\mathrm{TiO}_{2} / \mathrm{GO} / \mathrm{RGO}-\mathrm{TiO}_{2}$ hybrid film and structure diagram of SC. Reproduced with permission from Ref. [80]. Copyright 2017, Wiley-VCH. (d) Schematic diagram and digital photographs of the preparation process of RGO-GO-RGO foam. (e) (1) Cross-section SEM image of RGO-GO-RGO foam; (2) EDS of the GO and RGO parts; (3) SEM image of GO part; (4) SEM image of RGO part. (f) (1-3) The volumetric specific capacitances, energy density and cyclic stability of the SC at compression strain of $0 \%$ (black) and $90 \%$ (red), respectively; (4) the relationship between the compress-strain and relative specific capacitance. Reproduced with permission from Ref. [81]. Copyright 2017, the Royal Society of Chemistry.

sembly using the filtration method, as shown in Fig. 6a, b. This preparation strategy is easily manipulated with universality. The introduction of $\mathrm{TiO}_{2}$, which can generate transition electrons under ultraviolet (UV) radiation, not only effectively promotes GO reduction but also hinders the accumulation of GO, providing a large number of ion transport channels. Fig. $6 c$ displays the SEM image of the prepared sandwich construction SC, with the thickness of each layer precisely controlled by the amount of dispersion during the filtration process. The result shows the specific capacitance of $56 \mathrm{mF} \mathrm{cm}^{-2}$ $\left(237 \mathrm{~F} \mathrm{~cm}^{-3}\right)$ at a scan rate of $2 \mathrm{mV} \mathrm{s}^{-1}$, energy density of $16 \mathrm{~mW} \mathrm{~h} \mathrm{~cm}^{-3}$ at a power density of $1.8 \mathrm{~W} \mathrm{~cm}^{-3}$ for a voltage window of $0.8 \mathrm{~V}$. However, the layered structure of membrane-like SCs limits the diffusion of ions and increases the diffusion resistance, resulting in poor rate performance.

In addition to $\mathrm{UV}$ radiation of RGO, laser reduction is also an effective strategy. Shao et al. [81] prepared the RGO-based SC by laser irradiation of the foam-supported 3D GO top and bottom surfaces, as shown in Fig. 6d. The research feature is that the specific capacitance and energy density of the SC can be controlled by a certain stress compression device because of the MF as the middle separator layer. The cross-section SEM image and energy spectrum image of the RGO-GO-RGO sandwich structure are displayed in Fig. 6e $(1,2)$. The average thickness of the electrode layer and separator layer are $680 \mu \mathrm{m}$, and the $\mathrm{O} / \mathrm{C}$ ratio of $\mathrm{RGO}$ in the laser reduction region is significantly lower than that in the non-reduction region, indicating that laser reduction is an effective means of reducing GO. The SEM images show that there is no significant damage or change in the structure skeleton before and after laser reduction (Fig. 6e $(3,4)$ ). Because the electrode material structure changed from porous $3 \mathrm{D}$ to $2 \mathrm{D}$ plane during the compression of device, the elec- 
trode material not only formed a dense conductive pathway, but also shortened the ion diffusion pathway by close contact with the separator, resulting in a sharp decline in resistance and improved electrochemical performance. Fig. $6 \mathrm{f}$ shows the volumetric specific capacitance, energy density and cyclic stability of the SC under $90 \%$ strain are all better than that without strain, and with the increase of compression degree, the relative specific capacitance increases gradually.

Moreover, the synthesis of all-in-one SC by chemical metal reduction of GO has also been reported [82]. Allin-one integrated flexible electrode and separator coupled with an aqueous/organic solution electrolyte device is called an all-in-one FSC instead of an all-solid-state (quasi-solid-state) all-in-one SC due to the use of liquid electrolyte. Currently, this type of SCs is prone to leakage due to the liquid electrolytes during the application, and thus encapsulation technology to solve the security problem is still a thorny problem. The application of allsolid-state all-in-one SCs in flexible electronic equipment has better prospect [83].

\section{$1 D$ ALL-IN-ONE SCs}

Fiber SCs are more suitable for flexible wearable electronics than 2D planar SCs. For example, it is easier to knit, knot and form other different shapes [84]. Macroscopically, the $1 \mathrm{D}$ structure usually varies in diameter from microns to millimeters, characterized by light weight, high flexibility and small volume [85]. From the perspective of assembly structure, fiber SCs can be divided into coaxial-fiber, twisted-fiber, parallel-fiber, coaxial-helix-fiber, consecutive-fiber and all-in-one-fiber SCs [86], as shown in Fig. 7a-f. Various types of fiber SCs have their advantages and disadvantages. For instance, twisted-fiber SCs are assembled by twisting two or more individual fibers with porous membrane or quasi-solid electrolyte. Due to the insufficient contact area between the electrodes, not only the energy density is not satisfactory, but also the fatigue resistance is poor during the deformation process. Coaxial-fiber SCs are assembled layer by layer to integrate the electrode-electrolyte-electrode on the fiber substrate, reducing the interface resistance compared with the twisted-fiber SCs, but also face the problem of short circuit caused by interlayer slip. All-in-one fiber SCs are a micromachining technique that allows electrodes to be carved out of the fiber substrate. It can reasonably combine two electrodes and the separator on a single fiber. Here, we do not advocate that coaxialand twisted-fiber SCs be referred to as all-in-one SCs in the published literatures $[87,88]$. In this section, we mainly introduce the research status of the all-in-one SCs.

Graphene fiber is the preferred material for carbonbased SCs due to its advantages of high mechanical strength, excellent flexibility and outstanding electrical conductivity. The commonly used preparation methods are reduction of GO fibers by wet spinning, molding (hydrothermal), rotation or winding methods. For example, Hu et al. [89] reported the fabrication of RGOGO-RGO all-in-one integrated SC using laser regionspecific reduction of GO fiber, which is similar to the preparation of $2 \mathrm{D}$ MFSCs mentioned above. In this structure, the GO acts as separator. As an electrical insulating material, GO has a conductivity of only about $5 \times 10^{-6} \mathrm{~S} \mathrm{~cm}^{-1}$ due to a large amount of oxygen-containing functional groups on its surface and edge [82]. However, the conductivity of RGO fibers is lower than graphene in the range of about 7.80 to $1,110 \mathrm{~S} \mathrm{~cm}^{-1}$, which is also in response to the fact that most RGO fiber electrodes exhibit pseudocapacitive electrochemical behavior due to the presence of partial oxygen-containing functional groups that have not been adequately reduced [86].

In order to solve the unsatisfactory capacitance and energy density of the carbon-based electrode SCs, laserinduced pseudo-capacitance materials are also reported. Zhou et al. [90] reported the fabrication of flexible SSC (FSSC) using laser scribing to carve the surface of PI fiber impregnated with active materials. $\mathrm{Mn}, \mathrm{Mo}, \mathrm{S}$ ions were used as precursors of active electrode materials $\left(\mathrm{MoS}_{2}\right.$ and $\mathrm{MnS})$ to prepare bi-metal sulfide/graphene nanoribbon (GR) hybrid electrode $\left(\mathrm{MoS}_{2} / \mathrm{MnS} / \mathrm{GR}\right)$ under laser induction, as shown in Fig. $7 \mathrm{~g}$. The morphology and structure of the $\mathrm{MoS}_{2} / \mathrm{MnS} / \mathrm{GR}$ electrode are shown in Fig. 7h. The width and thickness of GR are about 105 and $2.83 \mathrm{~nm}$, respectively. $\mathrm{MoS}_{2}$ and $\mathrm{MnS}$ have been successfully modified on the surface of GR with a size of about $15-30 \mathrm{~nm}$. The high-resolution transmission electron microscopy (TEM) indicates that the interplanar spacing of $\mathrm{MoS}_{2}, \mathrm{MnS}$ and GR is $0.62,0.30$, and $0.34 \mathrm{~nm}$, respectively. The $\mathrm{MoS}_{2} / \mathrm{MnS} / \mathrm{GR}$ FSSC achieved an areal specific capacitance of $50.2 \mathrm{mF} \mathrm{cm}^{-2}$ and length specific capacitance of $401.7 \mu \mathrm{F} \mathrm{cm}^{-1}$ at a current density of $50 \mu \mathrm{A} \mathrm{cm}^{-2}$, and a high energy density of $7.0 \mu \mathrm{W} \mathrm{h} \mathrm{cm}{ }^{-2}$ at the power density of $49.9 \mu \mathrm{W} \mathrm{cm}{ }^{-2}$ (Fig. 7i). After bending for 1,000 cycles, the GCD curves show a small $I R$ drop of $0.05 \mathrm{~V}$ (Fig. 7j), indicating that the all-in-one FSSC has excellent bending durability and provides a basis for realizing braided Kevlar fabric for the application in wearable electronics, with the realistic application of the all-in-one SC by four devices in series shown in 


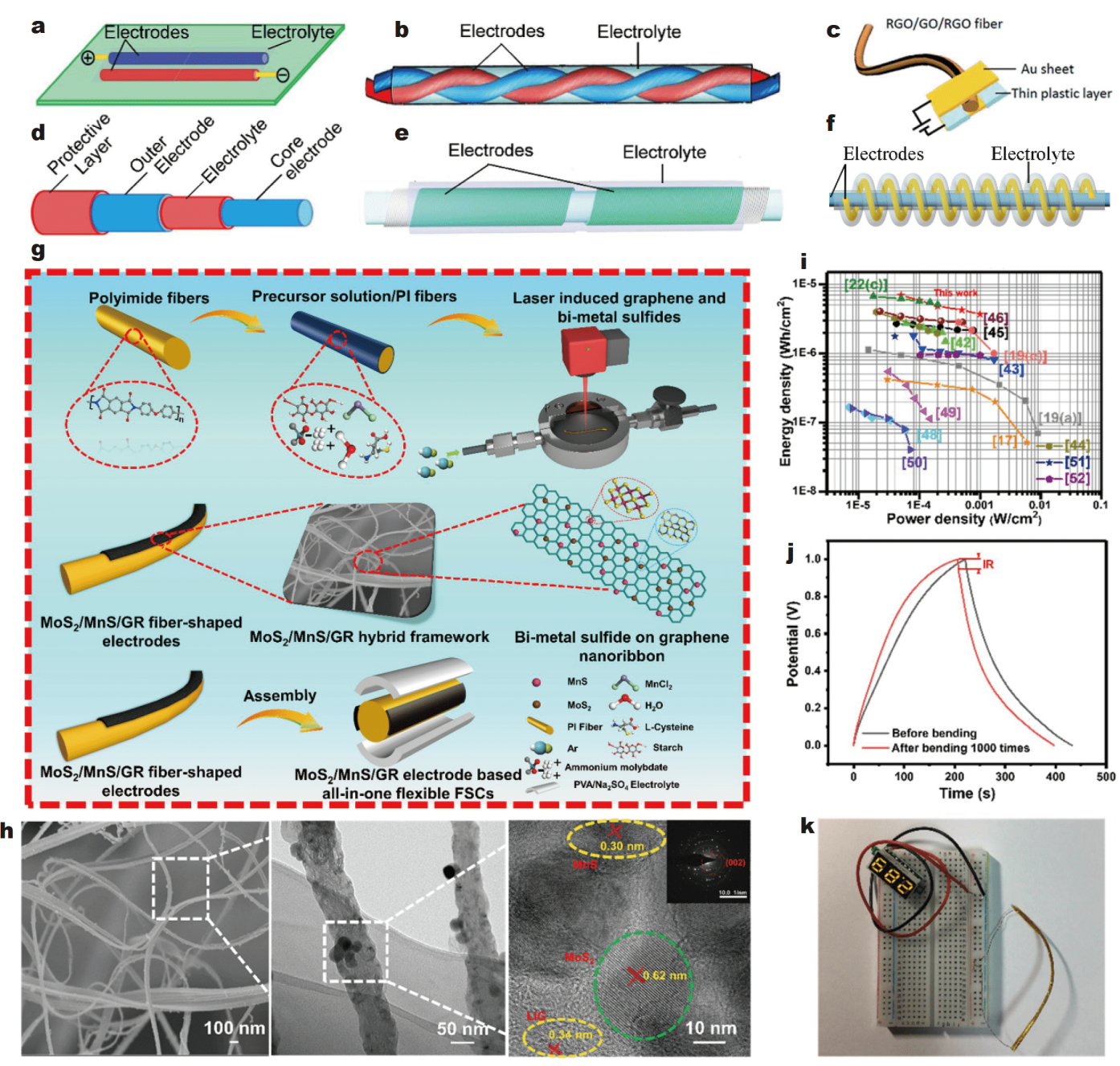

Figure 7 Fiber SCs with different assembly structures (a-f). (a) Parallel. Reproduced with permission from Ref. [91]. Copyright 2017, Elsevier. (b) Twisting. Reproduced with permission from Ref. [92]. Copyright 2013, the Royal Society of Chemistry. (c) All-in-one. Reproduced with permission from Ref. [89]. Copyright 2014, the Royal Society of Chemistry. (d) Coaxial. Reproduced with permission from Ref. [91]. (e) Consecutive. Reproduced with permission from Ref. [93]. Copyright 2014, Wiley-VCH. (f) Coaxial-helix. (g) Schematic diagram of the preparation of MoS $2 / \mathrm{MnS}_{\mathrm{GR}}$ based allin-one FSC. (h) SEM and TEM images of $\mathrm{MoS}_{2} / \mathrm{MnS} / \mathrm{GR}$ electrode. (i) Energy density and power density of $\mathrm{MoS}_{2} / \mathrm{MnS} / \mathrm{GR}$ FSSCs (the reference numbers refer to Ref. [90]). (j) GCD curves of the $\mathrm{MoS}_{2} / \mathrm{MnS} / \mathrm{GR}$ FSSC before and after 1000 times bending (current density of $100 \mu \mathrm{A} \mathrm{cm}{ }^{-2}$ ). $(\mathrm{k})$ Realistic application of the all-in-one SC by four devices in series. Reproduced with permission from Ref. [90]. Copyright 2019, Elsevier.

Fig. 7k.

\section{CHALLENGES AND OUTLOOK}

All-in-one SCs are expected to be used in the field of flexible electronics. However, the following challenges need to be figured out.

(1) The durability of FSCs to store energy is of great importance in practical applications, and it is a challenge to judge whether they can be applied in practice because few studies have provided the self-discharge phenomenon data of SCs. Although the self-discharge phenomenon is inevitable in the energy storage, researchers can design electrodes, electrolytes and separators to suppress the selfdischarge phenomenon as much as possible. The selfdischarge mechanism of SCs mainly includes ion diffusion redistribution, Faraday reaction and leakage resistance. Self-discharge current (leakage current) is a parameter related to the equivalent parallel resistance inside the structure, and both leakage current and selfdischarge affect the discharge efficiency of the SC. Some studies suggest that the phenomenon of self-discharge is particularly serious in all-solid SCs, in which the opencircuit voltage drops to half of the original level within tens of minutes [94]. It has also been shown that the self- 
discharging performance of PAM-based hydrogel electrolytes is slower than that of PVA-based hydrogels [95]. In order to more comprehensively express the performance of SC devices, it is necessary to study the selfdischarge phenomenon.

(2) In order to develop a variety of FSCs that can be applied in different fields, it is necessary to understand the requirements of practical applications. For example, as a power source such as a human knuckle sensor, the tensile strain only needs to be less than $63 \%$, so there is no need to go beyond $200 \%$ of the stretchability [96], and the pursuit of high values is inferior to understanding the parameters of design requirements. Dynamic capacitance testing is more practical than static testing in the application, and in addition to static proof of stretchability, it is also important to know the times that the device can be stretched and the corresponding strain residue. In general, the strain rate has a certain influence on the degree of reversible recovery and stress residue of the device, but the reported studies seldom mention this. In fact, the design of SCs for specific needs has begun. For instance, in order to adapt to the special environment (high and low temperature), the low-temperature-resistant all-inone SC with ideal capacitance at $-36^{\circ} \mathrm{C}$ has been developed [97], and an all-temperature FSC was manufactured to accommodate a wider range of temperatures $(-50$ to $\left.90^{\circ} \mathrm{C}\right)[98]$.

(3) It is still a challenge to systematically study the rate performance of SCs. In order to claim that the SC has excellent rate performance, the GCD is tested under a group of relatively small current densities. However, it is not convincing enough to prove that it has excellent rate capacity. We suggest testing the device under a group of relatively large current densities. In the last decade, most of the published papers suggested 2D materials have better rate performance than the non-2D materials due to solid state diffusion with a short time. However, different points of view are recently put forward through comprehensive analysis of a large number of previous experimental data and related models showing that electrolyte ions have low ion mobility in $2 \mathrm{D}$ materials [99].

(4) Although hydrogel-based FSCs have demonstrated excellent mechanical properties, especially PAM-based and PAA-based hydrogels, it remains challenging to modify the molecular structure of hydrogel so that it has a strong interaction with water molecules to lock up the water and slow volatilization. More studies are needed to solve the problem of water loss in air or encapsulation during hydrogel placement. The water content of the hydrogel balances ionic conductivity and mechanical strength. The inherent structural defects of PVA, such as the limited number of lateral hydroxyl groups, insufficient hydrophilicity, long carbon chains, lead to the weak interaction between the framework polymer and the water medium, which further results in rapid evaporation of water, sharp decline in ionic conductivity, and device performance plummeting or failure [28].

(5) It is still a long-term challenge to pursue FSCs with high volumetric energy density (which is suitable for practical applications) and stretchable properties simultaneously. Stretchable all-in-one SCs are usually hydrogel-based SCs with an inherently cumbersome volume, and with polydimethylsiloxane (PDMS) packaging, the energy density of the entire device is not satisfactory. At the same time, the tensile strength of the encapsulated SC will largely depend on the tensile strength of PDMS, in which case, it will be futile to blindly pursue the electrolyte or electrode with ultra-high tensile strength.

Despite these challenges, flexible all-in-one SCs are promising as energy storage devices for flexible electronics. For instance, coaxial fiber SCs are used as power supply devices for strain sensors in response to the complex and variable movement of human body [87]. Research on integrated SCs with multiple functions has begun to develop, such as transparancy, self-charge, electrochromic properties, shape memory, and so on. For example, the all-in-one shape-adaptive self-charging SC has been designed for power package in wearable electronics [100].

\section{CONCLUSIONS}

The all-in-one integrated SCs not only have a slight resistance at the interface between the electrode and separator or electrolyte, but also can maintain excellent electrochemical performance during various deformations. Therefore, it is more promising to provide energy storage devices for wearable and portable electronics. The recent advances and preparation methods of all-in-one integrated FSCs are reviewed. 2D all-in-one SCs are assembled with gel electrolyte as the structural matrix, which exhibit unique tensile and self-healing properties but with large volume. Preparation of excellent hydrogel electrolytes is important to obtain the device with high performance. 2D all-in-one SCs are assembled on the basis of separator (non-hydrogel) exhibiting high volumetric energy density which is a relatively simple fabrication process but generally does not exhibit tensile or self-healing properties. 1D all-in-one SCs are easier to be 
woven into the Kevlar fabric for wearable applications, but typically have low energy density and require micromachining technology to process electrodes on the fiber matrix. However, the main factor affecting the largescale development of this kind of SCs is the lack of popular fiber surface micromachining technology. The bridge between the basic research and the practical industrial application of FSCs needs to be built.

\section{Received 9 April 2020; accepted 1 June 2020; published online 6 August 2020}

1 Li L, Lou Z, Chen D, et al. Recent advances in flexible/stretchable supercapacitors for wearable electronics. Small, 2018, 14: 1702829

2 Liu YM, Merlet C, Smit B. Carbons with regular pore geometry yield fundamental insights into supercapacitor charge storage. ACS Cent Sci, 2019, 5: 1813-1823

$3 \mathrm{Xu} \mathrm{Z}$, Wang T, Wang L, et al. Aniline-grafting graphene oxide/ polyaniline composite prepared via interfacial polymerization with high capacitive performance. Int J Energy Res, 2019, 43: 7693

4 Wang J, Lou H, Meng J, et al. Stretchable energy storage e-skin supercapacitors and body movement sensors. Sens Actuat BChem, 2020, 305: 127529

5 Chen X, Villa NS, Zhuang Y, et al. Stretchable supercapacitors as emergent energy storage units for health monitoring bioelectronics. Adv Energy Mater, 2020, 10: 1902769

6 Park H, Kim JW, Hong SY, et al. Dynamically stretchable supercapacitor for powering an integrated biosensor in an all-inone textile system. ACS Nano, 2019, 13: 10469-10480

7 Hsiao C, Lee C, Tai N. Biomass-derived three-dimensional carbon framework for a flexible fibrous supercapacitor and its application as a wearable smart textile. RSC Adv, 2020, 10: 69606972

8 Xue Q, Sun J, Huang Y, et al. Recent progress on flexible and wearable supercapacitors. Small, 2017, 13: 1701827

9 Ji C, Mi H, Yang S. Latest advances in supercapacitors: From new electrode materials to novel device designs. Chin Sci Bull, 2018, 64: 9-34

10 Wang X, Yang C, Jin J, et al. High-performance stretchable supercapacitors based on intrinsically stretchable acrylate rubber/ MWCNTs@conductive polymer composite electrodes. J Mater Chem A, 2018, 6: 4432-4442

11 Liu Q, Zang L, Qiao X, et al. Compressible all-in-one supercapacitor with adjustable output voltage based on polypyrrolecoated melamine foam. Adv Electron Mater, 2019, 5: 1900724

12 Wang Y, Gong S, Dong D, et al. Self-assembled gold nanorime mesh conductors for invisible stretchable supercapacitors. Nanoscale, 2018, 10: 15948-15955

13 Wang Y, Ding Y, Guo X, et al. Conductive polymers for stretchable supercapacitors. Nano Res, 2019, 12: 1978-1987

14 Lv Z, Tang Y, Zhu Z, et al. Honeycomb-lantern-inspired 3D stretchable supercapacitors with enhanced specific areal capacitance. Adv Mater, 2018, 30: 1805468

15 He S, Qiu L, Wang L, et al. A three-dimensionally stretchable high performance supercapacitor. J Mater Chem A, 2016, 4: 14968-14973

16 Xie Y, Liu Y, Zhao Y, et al. Stretchable all-solid-state super- capacitor with wavy shaped polyaniline/graphene electrode. J Mater Chem A, 2014, 2: 9142-9149

17 Lee H, Lee G, Yun J, et al. Facile fabrication of a fully biodegradable and stretchable serpentine-shaped wire supercapacitor. Chem Eng J, 2019, 366: 62-71

18 Wang S, Liu N, Su J, et al. Highly stretchable and self-healable supercapacitor with reduced graphene oxide based fiber springs. ACS Nano, 2017, 11: 2066-2074

19 Zhang R, Yan K, Palumbo A, et al. A stretchable and bendable allsolid-state pseudocapacitor with dodecylbenzenesulfonate-doped polypyrrole-coated vertically aligned carbon nanotubes partially embedded in PDMS. Nanotechnology, 2019, 30: 095401

20 Jeong HT. Electrochemical performances of semi-transparent and stretchable supercapacitor composed of nanocarbon materials. Carbon Lett, 2020, 30: 55-61

21 Tang Q, Chen M, Wang G, et al. A facile prestrain-stick-release assembly of stretchable supercapacitors based on highly stretchable and sticky hydrogel electrolyte. J Power Sources, 2015, 284: 400-408

22 Wang B, Song W, Gu P, et al. A stretchable and hydrophobic polypyrrole/knitted cotton fabric electrode for all-solid-state supercapacitor with excellent strain capacitance. Electrochim Acta, 2019, 297: 794-804

23 Qi F, Zhao C, Wang C, et al. Polyaniline electrochemically deposited on tailored metal mesh for dynamically stretchable supercapacitors. J Electrochem Soc, 2019, 166: A3932-A3939

24 Zhang S, Pan N. Supercapacitors performance evaluation. Adv Energy Mater, 2015, 5: 1401401

25 Li K, Zhang J. Recent advances in flexible supercapacitors based on carbon nanotubes and graphene. Sci China Mater, 2017, 61: 210-232

26 Wang X, Ding W, Li H, et al. Unveiling highly ambient-stable multilayered $1 \mathrm{~T}-\mathrm{MoS}_{2}$ towards all-solid-state flexible supercapacitors. J Mater Chem A, 2019, 7: 19152-19160

27 Shao Y, El-Kady MF, Sun J, et al. Design and mechanisms of asymmetric supercapacitors. Chem Rev, 2018, 118: 9233-9280

28 Wang Z, Li H, Tang Z, et al. Hydrogel electrolytes for flexible aqueous energy storage devices. Adv Funct Mater, 2018, 28: 1804560

29 Wang K, Zhang X, Li C, et al. Chemically crosslinked hydrogel film leads to integrated flexible supercapacitors with superior performance. Adv Mater, 2015, 27: 7451-7457

$30 \mathrm{Hu}$ R, Wang Y, Zhao J, et al. Fabrication of stretchable multielement composite for flexible solid-state electrochemical capacitor application. Chem Eng J, 2019, 361: 109-116

31 Chen Z, Yang Y, Ma Z, et al. All-solid-state asymmetric supercapacitors with metal selenides electrodes and ionic conductive composites electrolytes. Adv Funct Mater, 2019, 29: 1904182

32 Guo Y, Zheng K, Wan P. A flexible stretchable hydrogel electrolyte for healable all-in-one configured supercapacitors. Small, 2018, 14: 1704497

33 Shih CC, Lin YC, Gao M, et al. A rapid and green method for the fabrication of conductive hydrogels and their applications in stretchable supercapacitors. J Power Sources, 2019, 426: 205-215

34 Chen CR, Qin H, Cong HP, et al. A highly stretchable and realtime healable supercapacitor. Adv Mater, 2019, 31: 1900573

35 Wang Y, Chen F, Liu Z, et al. A highly elastic and reversibly stretchable all-polymer supercapacitor. Angew Chem Int Ed, 2019, 58: 15707-15711

36 Jin $\mathrm{X}$, Sun G, Yang H, et al. A graphene oxide-mediated poly- 
electrolyte with high ion-conductivity for highly stretchable and self-healing all-solid-state supercapacitors. J Mater Chem A, 2018, 6: 19463-19469

37 Cao C, Li Y. Highly stretchable calcium ion/polyacrylic acid hydrogel prepared by freezing-thawing. J Mater Sci, 2020, 55: 53405348

38 Wang T, Zhang Y, Liu Q, et al. A self-healable, highly stretchable, and solution processable conductive polymer composite for ultrasensitive strain and pressure sensing. Adv Funct Mater, 2018, 28: 1705551

39 Huang Y, Zhong M, Huang Y, et al. A self-healable and highly stretchable supercapacitor based on a dual crosslinked polyelectrolyte. Nat Commun, 2015, 6: 10310

40 Mao J, Zhao C, Li Y, et al. Highly stretchable, self-healing, and strain-sensitive based on double-crosslinked nanocomposite hydrogel. Compos Commun, 2020, 17: 22-27

41 Jia R, Du H, Zhang X, et al. Stretchable and compressible supercapacitor with polyaniline on hydrogel electrolyte. J Electrochem Soc, 2018, 165: A3792-A3798

42 Shi Y, Zhang Y, Jia L, et al. Stretchable and self-healing integrated all-gel-state supercapacitors enabled by a notch-insensitive supramolecular hydrogel electrolyte. ACS Appl Mater Interfaces, 2018, 10: 36028-36036

43 Zhao Y, Alsaid Y, Yao B, et al. Wood-inspired morphologically tunable aligned hydrogel for high-performance flexible all-solidstate supercapacitors. Adv Funct Mater, 2020, 30: 1909133

44 Yin BS, Zhang SW, Ke K, et al. Advanced deformable all-in-one hydrogel supercapacitor based on conducting polymer: Toward integrated mechanical and capacitive performance. J Alloys Compd, 2019, 805: 1044-1051

45 Zhang B, Gao Z, Gao G, et al. Highly mechanical and fatigueresistant double network hydrogels by dual physically hydrophobic association and ionic crosslinking. Macromol Mater Eng, 2018, 303: 1800072

46 Wang M, Fan L, Qin G, et al. Flexible and low temperature resistant semi-IPN network gel polymer electrolyte membrane and its application in supercapacitor. J Membrane Sci, 2020, 597: 117740

47 Tuncaboylu DC, Sari M, Oppermann W, et al. Tough and selfhealing hydrogels formed via hydrophobic interactions. Macromolecules, 2011, 44: 4997-5005

48 Zheng SY, Liu C, Jiang L, et al. Slide-ring cross-links mediated tough metallosupramolecular hydrogels with superior self-recoverability. Macromolecules, 2019, 52: 6748-6755

49 Bai Y, Liu R, Wang Y, et al. High ion transport within a freezecasted gel film for high-rate integrated flexible supercapacitors. ACS Appl Mater Interfaces, 2019, 11: 43294-43302

50 Wang F, Wu X, Yuan X, et al. Latest advances in supercapacitors: From new electrode materials to novel device designs. Chem Soc Rev, 2017, 46: 6816-6854

51 Jeong HT, Du JF, Kim YR, et al. Electrochemical performances of highly stretchable polyurethane (PU) supercapacitors based on nanocarbon materials composites. J Alloys Compd, 2019, 777: $67-72$

52 Liang X, Zhao L, Wang Q, et al. A dynamic stretchable and selfhealable supercapacitor with a CNT/graphene/PANI composite film. Nanoscale, 2018, 10: 22329-22334

53 Liu S, Lin Y, Wei Y, et al. A high performance self-healing strain sensor with synergetic networks of poly( $\varepsilon$-caprolactone) microspheres, graphene and silver nanowires. Compos Sci Tech, 2017,
146: 110-118

54 Yang J, Yu X, Sun X, et al. Polyaniline-decorated supramolecular hydrogel with tough, fatigue-resistant, and self-healable performances for all-in-one flexible supercapacitors. ACS Appl Mater Interfaces, 2020, 12: 9736-9745

55 Liu F, Wang J, Pan Q. An all-in-one self-healable capacitor with superior performance. J Mater Chem A, 2018, 6: 2500-2506

56 Zeng J, Dong L, Sha W, et al. Highly stretchable, compressible and arbitrarily deformable all-hydrogel soft supercapacitors. Chem Eng J, 2020, 383: 123098

57 Liao H, Zhou F, Zhang Z, et al. A self-healable and mechanical toughness flexible supercapacitor based on polyacrylic acid hydrogel electrolyte. Chem Eng J, 2019, 357: 428-434

$58 \mathrm{Hu} \mathrm{M}$, Wang J, Liu J, et al. An intrinsically compressible and stretchable all-in-one configured supercapacitor. Chem Commun, 2018, 54: 6200-6203

59 Huang $\mathrm{Y}$, Zhong M, Shi F, et al. An intrinsically stretchable and compressible supercapacitor containing a polyacrylamide hydrogel electrolyte. Angew Chem Int Ed, 2017, 56: 9141-9145

60 Wang H, Dai L, Chai D, et al. Recyclable and tear-resistant all-inone supercapacitor with dynamic electrode/electrolyte interface. J Colloid Interface Sci, 2020, 561: 629-637

61 Zang L, Liu Q, Qiu J, et al. Design and fabrication of an all-solidstate polymer supercapacitor with highly mechanical flexibility based on polypyrrole hydrogel. ACS Appl Mater Interfaces, 2017, 9: 33941-33947

62 Hyeon SE, Seo JY, Bae JW, et al. Faradaic reaction of dual-redox additive in zwitterionic gel electrolyte boosts the performance of flexible supercapacitors. Electrochim Acta, 2019, 319: 672-681

63 Yadav N, Yadav N, Singh MK, et al. Nonaqueous, redox-active gel polymer electrolyte for high-performance supercapacitor. Energy Technol, 2019, 7: 1900132

64 Guo C, Zhang Y, Zeng T, et al. High-performance asymmetric supercapacitors using holey graphene electrodes and redox electrolytes. Carbon, 2020, 157: 298-307

65 Jinisha B, Anilkumar KM, Manoj M, et al. Solid-state supercapacitor with impressive performance characteristics, assembled using redox-mediated gel polymer electrolyte. J Solid State Electrochem, 2019, 23: 3343-3353

66 Fang J, Miao X, Zhang X, et al. Enhancing the capacity of activated carbon electrodes by a redox mediator pair for the fabrication of flexible asymmetric solid-state supercapacitors. J Power Sources, 2019, 418: 24-32

67 Cevik E, Bozkurt A, Hassan M, et al. Redox-mediated poly(2acrylamido-2-methyl-1-propanesulfonic acid)/ammonium molybdate hydrogels for highly effective flexible supercapacitors. ChemElectroChem, 2019, 6: 2876-2882

68 Cevik E, Bozkurt A, Dirican M, et al. High performance flexible supercapacitors including redox active molybdate incorporated poly(vinylphosphonic acid) hydrogels. Int J Hydrogen Energy, 2020, 45: 2186-2194

69 Cevik E, Bozkurt A. Design of high-performance flexible symmetric supercapacitors energized by redox-mediated hydrogels including metal-doped acidic polyelectrolyte. Int J Energy Res, 2020, 44: 4309-4320

70 Park J, Yoo YE, Mai L, et al. Rational design of a redox-active nonaqueous electrolyte for a high-energy-density supercapacitor based on carbon nanotubes. ACS Sustain Chem Eng, 2019, 7: 7728-7735

71 Gao T, Zhou Z, Yu J, et al. All-in-one compact architecture to- 
ward wearable all-solid-state, high-volumetric-energy-density supercapacitors. ACS Appl Mater Interfaces, 2018, 10: 2383423841

72 Wang N, Han G, Xiao Y, et al. Polypyrrole/graphene oxide deposited on two metalized surfaces of porous polypropylene films as all-in-one flexible supercapacitors. Electrochim Acta, 2018, 270: $490-500$

73 El-Kady MF, Kaner RB. Scalable fabrication of high-power graphene micro-supercapacitors for flexible and on-chip energy storage. Nat Commun, 2013, 4: 1475

74 Abdolhosseinzadeh S, Schneider R, Verma A, et al. Turning trash into treasure: Additive free MXene sediment inks for screenprinted micro-supercapacitors. Adv Mater, 2020, 32: 2000716

75 Zhang CJ, Kremer MP, Seral-Ascaso A, et al. Stamping of flexible, coplanar micro-supercapacitors using mxene inks. Adv Funct Mater, 2018, 28: 1705506

76 Zhang C, McKeon L, Kremer MP, et al. Additive-free Mxene inks and direct printing of micro-supercapacitors. Nat Commun, 2019, 10: 1795

77 Zheng S, Zhang CJ, Zhou F, et al. Ionic liquid pre-intercalated Mxene films for ionogel-based flexible micro-supercapacitors with high volumetric energy density. J Mater Chem A, 2019, 7: 9478-9485

78 Gao J, Shao C, Shao S, et al. Laser-assisted large-scale fabrication of all-solid-state asymmetrical micro-supercapacitor array. Small, 2018, 14: 1801809

79 Wang Y, Wang Y, Zhang P, et al. Laser-induced freestanding graphene papers: A new route of scalable fabrication with tunable morphologies and properties for multifunctional devices and structures. Small, 2018, 14: 1802350

$80 \mathrm{Du} \mathrm{J}$, Zheng C, Lv W, et al. A three-layer all-in-one flexible graphene film used as an integrated supercapacitor. Adv Mater Interfaces, 2017, 4: 1700004

81 Shao C, Xu T, Gao J, et al. Flexible and integrated supercapacitor with tunable energy storage. Nanoscale, 2017, 9: 12324-12329

82 Wang X, Wang R, Zhao Z, et al. Controllable spatial engineering of flexible all-in-one graphene-based supercapacitors with various architectures. Energy Storage Mater, 2019, 23: 269-276

83 Kang DA, Kim K, Karade SS, et al. High-performance solid-state bendable supercapacitors based on PEGBEM-g-PAEMA graft copolymer electrolyte. Chem Eng J, 2020, 384: 123308

84 Yang C, Pan Q, Jia Q, et al. Multifunctional microporous activated carbon nanotubes anchored on graphite fibers for highstrength and high-rate flexible all-solid-state supercapacitors. Appl Surf Sci, 2020, 502: 144423

85 Liu L, Niu Z, Chen J. Unconventional supercapacitors from nanocarbon-based electrode materials to device configurations. Chem Soc Rev, 2016, 45: 4340-4363

86 Zhai S, Karahan HE, Wang C, et al. 1D supercapacitors for emerging electronics: Current status and future directions. Adv Mater, 2020, 32: 1902387

87 Pan Z, Yang J, Li L, et al. All-in-one stretchable coaxial-fiber strain sensor integrated with high-performing supercapacitor. Energy Storage Mater, 2020, 25: 124-130

88 Yu J, Zhou J, Yao P, et al. A stretchable high performance all-inone fiber supercapacitor. J Power Sources, 2019, 440: 227150

$89 \mathrm{Hu} \mathrm{Y}$, Cheng H, Zhao F, et al. All-in-one graphene fiber supercapacitor. Nanoscale, 2014, 6: 6448-6451

90 Zhou C, Hong M, Yang Y, et al. Laser-induced bi-metal sulfide/ graphene nanoribbon hybrid frameworks for high-performance all-in-one fiber supercapacitors. J Power Sources, 2019, 438: 227044

91 Meng F, Li Q, Zheng L. Flexible fiber-shaped supercapacitors: design, fabrication, and multi-functionalities. Energy Storage Mater, 2017, 8: 85-109

92 Cai Z, Li L, Ren J, et al. Flexible, weavable and efficient microsupercapacitor wires based on polyaniline composite fibers incorporated with aligned carbon nanotubes. J Mater Chem A, 2013, 1: 258-261

93 Chen X, Lin H, Deng J, et al. Electrochromic fiber-shaped supercapacitors. Adv Mater, 2014, 26: 8126-8132

94 Verma ML, Minakshi M, Singh NK. Synthesis and characterization of solid polymer electrolyte based on activated carbon for solid state capacitor. Electrochim Acta, 2014, 137: 497-503

$95 \mathrm{Li} \mathrm{H}, \mathrm{Lv} \mathrm{T}, \mathrm{Li} \mathrm{N}$, et al. Ultraflexible and tailorable all-solid-state supercapacitors using polyacrylamide-based hydrogel electrolyte with high ionic conductivity. Nanoscale, 2017, 9: 18474-18481

96 Qiu A, Li P, Yang Z, et al. A path beyond metal and silicon: polymer/nanomaterial composites for stretchable strain sensors. Adv Funct Mater, 2019, 29: 1806306

97 Wang Y, Yuan H, Zhu Y, et al. An all-in-one supercapacitor working at sub-zero temperatures. Sci China Mater, 2020, 63: 660-666

98 Lu C, Chen X. All-temperature flexible supercapacitors enabled by antifreezing and thermally stable hydrogel electrolyte. Nano Lett, 2020, 20: 1907-1914

99 Tian R, Breshears M, Horvath DV, et al. The rate performance of two-dimensional material-based battery electrodes may not be as good as commonly believed. ACS Nano, 2020, 14: 3129-3140

100 Guo H, Yeh MH, Lai YC, et al. All-in-one shape-adaptive selfcharging power package for wearable electronics. ACS Nano, 2016, 10: $10580-10588$

Acknowledgements This work was supported by the National Key Research and Development Program of China (2017YFB0406301), the State Key Laboratory of Electrical Insulation and Power Equipment (EIPE19210), the 111 Project of China (B14040) and the Fundamental Research Funds for the Central University.

Author contributions Guo T and Zhou D wrote the manuscript; Liu $\mathrm{W}$ and $\mathrm{Su} J$ participated in the discussion.

Conflict of interest The authors declare no conflict of interests.

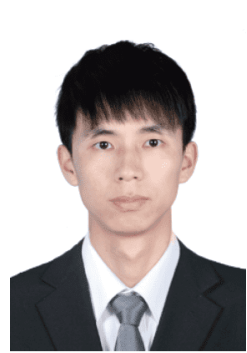

Tiezhu Guo received his bachelor degree from the Northwest Minzu University in 2015 and master degree from the South China University of Technology in 2018. He is a $\mathrm{PhD}$ candidate at Xi'an Jiaotong University. His research interests mainly focus on flexible supercapacitors. 


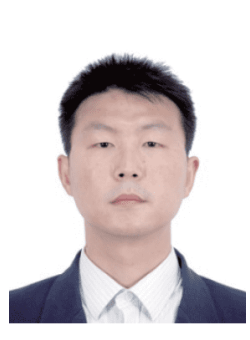

Di Zhou is currently a professor in the School of Electronic Science and Engineering, Xi'an Jiaotong University. $\mathrm{He}$ received his $\mathrm{PhD}$ degree majored in electronic science and technology under the guidance of Prof. Xi Yao, from Xi'an Jiaotong University in 2009. He worked as a research associate in Prof. Ian Reaney's group during 2015-2018 at the University of Sheffield. His research interests include microwave dielectric materials, low temperature co-fired ceramics technology (LTCC), energy storage capacitor materials, microwave absorption materials, and functional composite materials for high frequency/energy storage/microwave absorption. He is now an Associate Editor of Journal of the American Ceramic Society and a board member of Materials Research Bulletin.

\section{一体化柔性超级电容器的研究进展}

郭铁柱 $^{1}$, 周迪 ${ }^{1,2^{*}}$, 刘文风 ${ }^{2}$, 苏进展 ${ }^{3}$

摘要 个性化柔性电子产品的兴起推动了柔性超级电容器的快速 发展, 这得益于它的使用寿命长、充放电速度快和操作安全等特 点. 不同于传统的柔性超级电容器, 一体化柔性超级电容器具有低 界面电阻和更耐形变的特性, 因而在柔性电子领域中有更广阔的 应用前景. 本文简要总结了一维纤维状和二维平面状的一些典型 一体化超级电容器的制备方法和电化学性能. 首先介绍了传统柔 性超级电容器和一体化柔性超级电容器的基本概念以及性能评估 的主要参数, 随后介绍了具有不同功能特征(可拉伸、自愈合和可 压缩) 水凝胶基和非水凝胶基一体化柔性超级电容器的研究进展, 并讨论了一体化柔性超级电容器在发展中面临的挑战, 且对未来 的发展方向做出了展望. 